\title{
THE
}

1995

\section{Motion and Evolution of Binary Tropical Cyclones in a Coupled Atmosphere-Ocean Numerical Model}

\author{
Alexander I. Falkovich \\ Alexander P. Khain \\ Isaac Ginis \\ University of Rhode Island, iginis@uri.edu
}

Follow this and additional works at: https://digitalcommons.uri.edu/gsofacpubs

\author{
Citation/Publisher Attribution \\ Falkovich, A.I., A.P. Khain, and I. Ginis, 1995: Motion and Evolution of Binary Tropical Cyclones in a \\ Coupled Atmosphere-Ocean Numerical Model. Mon. Wea. Rev., 123, 1345-1363, doi: 10.1175/ \\ 1520-0493(1995)1232.0.CO;2. \\ Available at: https://doi.org/10.1175/1520-0493(1995)123<1345:MAEOBT>2.0.C0;2
}

This Article is brought to you for free and open access by the Graduate School of Oceanography at DigitalCommons@URI. It has been accepted for inclusion in Graduate School of Oceanography Faculty Publications by an authorized administrator of DigitalCommons@URI. For more information, please contact digitalcommons-group@uri.edu. 


\title{
Motion and Evolution of Binary Tropical Cyclomes in a Coupled Atmosphere-Ocean Numerical Mlodel
}

\author{
AleXANDER I. FALKovich* \\ Hydrometeorological Scientific Center, Moscow, Russian Federation \\ AleXander P. Khain \\ Institute of Earth Sciences, Hebrew University of Jerusalem, Givat Ram, Jerusalem, Israel \\ ISAAC GINIS \\ Graduate School of Oceanography, University of Rhode Island, Narragansett, Rhode Island
}

(Manuscript received 1 March 1994, in final form 10 October 1994)

\begin{abstract}
The interaction of binary tropical cyclones (TC) is investigated using a coupled TC-ocean movable nestedgrid model. The model consists of an eight-layer atmospheric model in the sigma coordinate system and a threelayer primitive equation ocean model. There are five meshes in the TC model. The outermost domain ( $3840 \mathrm{~km}$ $\times 3840 \mathrm{~km}$ ) is motionless. For the description of each TC in a TC pair, two telescopically nested meshes of finer resolution are used. The pair of the middle $(1600 \mathrm{~km} \times 1600 \mathrm{~km})$ and innermost $(800 \mathrm{~km} \times 800 \mathrm{~km})$ meshes move with the center of a corresponding TC. The space increments of the outermost domain and the middle and finest meshes are 160,80 , and $40 \mathrm{~km}$. The oceanic domain contains $107 \times 107$ grid points, with the spatial increment of $40 \mathrm{~km}$. In all numerical experiments a pair of equal strength axisymmetric vortices was located at different separation distances.

Experiments show that the rate of development of interacting TCs is different, mainly due to the difference in the velocities of TC movement. There is a "critical" separation distance between the centers of TCs, so that in case the separation distance is less than this critical value, attraction and merger of the TCs were observed. The critical separation distance depends on the structure of the vorticity field created by the binary TCs. Because of the changes in the structure of a TC during its life cycle the critical separation distance should also change. Two mechanisms related to the mutual vorticity advection and to the activity of irrotational velocity components seem to contribute to the attraction and repulsion of binary TCs.

The impact of the TC-ocean interaction on the evolution and trajectory of binary TCs is much stronger than in the case of a single TC. A decrease in TC strength is related not only to a TC response to seawater cooling caused by the TC itself but also to the crossing of the cold water wakes created both by the other TC and by the TC itself. A decrease in strength leads to a decrease in the mutual rotation velocity and, consequently, to a marked change in the trajectories of each of the interacting TCs. Changes in the structure of binary TCs caused by the TC-ocean interaction lead to an increase of the critical separation distance. Binary TCs cause seawater cooling over vast ocean areas and lead to the formation of a spotted sea surface temperature pattern.
\end{abstract}

\section{Introduction}

It is a well-established fact that some tropical cyclones, especially those developing in the western and eastern North Pacific, exist simultaneously within various separation distances (Fig. 1) (Gray 1979; Khain 1984). Nearly one-third of all tropical cyclones in the

\footnotetext{
* Current affiliation: National Meteorological Center, Development Division, Washington, D.C.
}

Corresponding author address: Dr. Alexander P. Khain, Department of Atmospheric Sciences, Institute of Earth Sciences, Givat Ram, Hebrew University of Jerusalem, Jerusalem 91904, Israel. western Pacific region during 1984 had a period of interaction with another cyclone (Neumann 1993). Interactions between two tropical cyclones frequently cause complicated storm motions (loops, sharp changes of direction, and translation speed). Large forecast errors are usually associated with an incorrect assessment of these situations (Brand 1970; Neumann 1981). Errors of $1850 \mathrm{~km}$ or more at $72 \mathrm{~h}$ may occur in these cases (Elsberry 1993). During the interaction, the circulation of one cyclone becomes the steering flow for the other cyclone, and vice versa. As a result, two vortices often rotate around an intermediate point. This effect in case of decrease of distance between cyclones is commonly known as the Fujiwhara effect (Fujiwhara 1921 ). Mutual rotation is usually a cyclonic one, but 


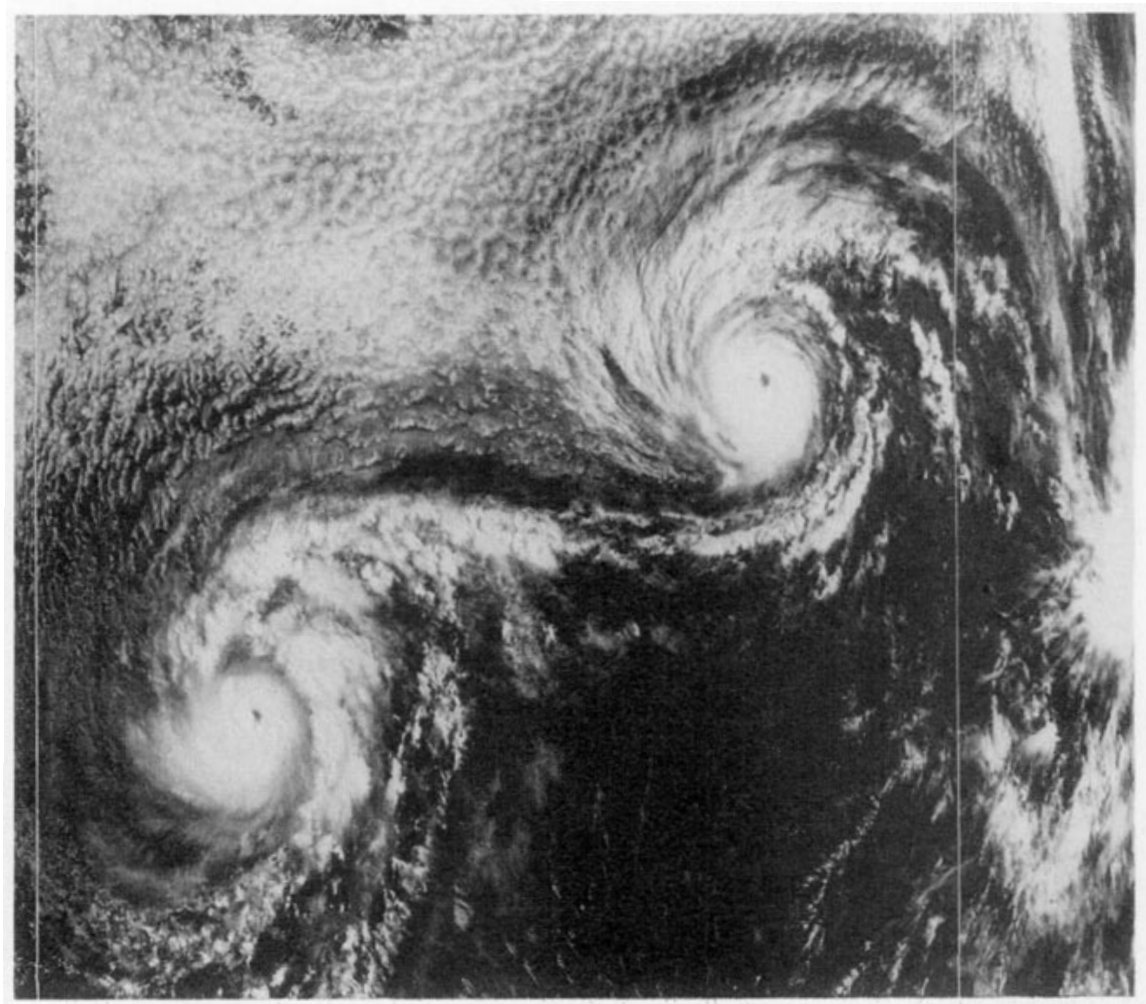

FIG. 1. Satellite picture of Tropical Cyclones Ione $\left(13.8^{\circ} \mathrm{N}, 142.2^{\circ} \mathrm{W}\right)$ and Kirsten (upper, right) 1749 UTC 24 August 1974. Distance between lines $10^{\circ}$. Tropical cyclones are located in the vicinity of Guam.

cases of anticyclonic rotation of a cyclone pair are also observed (Dong and Neumann 1983; Pokhil 1990). During the interaction period, binary vortices can merge or move away depending on the storm structures and intensities and the distance of separation. The literature on interaction of two tropical cyclones is rather limited. Theoretical and numerical studies were primarily performed by using nondivergent barotropic models (Khadekar and Rao 1971; DeMaria and Chan 1984; Pokhil et al. 1990; Chan 1993; Ritchie and Holland 1993). These studies were primarily concerned with mutual attraction or repulsion between binary vortices. Chang (1983) compared the interaction between vortex pairs in a barotropic model to those in a threedimensional baroclinic model in which a prescribed heating source was specified to maintain the tropical cyclone intensity. No mutual attraction was found between barotropic vortices in any experiments performed in which the initial separation distances were equal to $300 \mathrm{~km}$ or greater than $300 \mathrm{~km}$. In the baroclinic model, however, binary cyclones merged if they were initially separated by a distance of less than 1000 $\mathrm{km}$. Chang concluded that the divergent component of the winds in the baroclinic model was responsible for the mutual attraction of the vortex pair. DeMaria and Chan (1984) in their comments on Chang's (1983) results suggested that the interaction of a vortex pair can be explained from the vorticity advection point of view alone. Whether two vortices will merge or move away depends on the vorticity distribution of the combined system. According to DeMaria and Chan (1984),

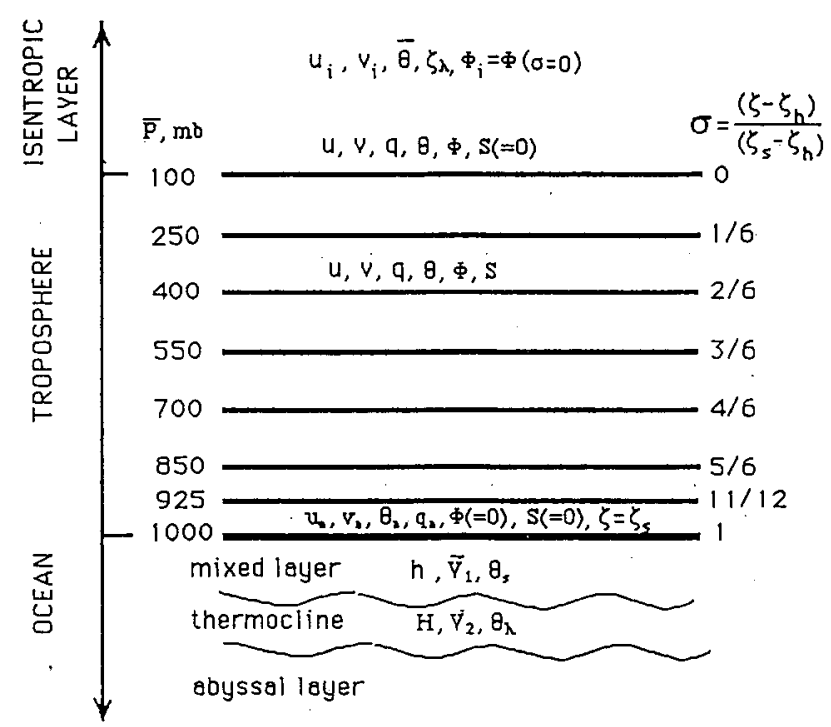

FIG. 2. Vertical structure of the atmosphere-ocean coupled model. 
two cyclonic vortices rotate cyclonically around each other due to the advection of the vorticity field associated with one vortex by the tangential winds of another vortex. The interaction of the tangential wind and vorticity fields can lead to either a decrease or an increase of the separation distance, depending on the sign of the vorticity gradient of the one vortex at the center of another one. DeMaria and Chan (1984) demonstrated that merger occurred (did not occur) if the separation is less (more) that a "critical" distance $d_{\mathrm{cr}}$. They assumed that $d_{\mathrm{cr}}$ is equal to the distance from the vortex center to the minimum of the radial vorticity profile $r_{c}$. DeMaria and Chan explained the absence of mutual attraction of barotropic vortices in Chang's simulations by the fact that the initial separations of the vortices was greater than $r_{c}$. Pokhil et al. (1990) showed that the critical separation distance depends on the initial tangential wind profiles in binary vortices. They used two types of radial distribution of the tangential velocity. In both cases the critical separation distance was about 1.5 times greater than the values of $r_{c}$ predicted by DeMaria and Chan (1984).

All studies discussed above were conducted on an $f$ plane and without a background flow. Recently Chan (1993) performed numerical simulations of binary vortices interaction on the beta plane with and without background flow using a nondivergent barotropic model. He concluded that the two vortices can be considered as a single system that moves toward the northwest due to the beta effect while they interact with each other. The interaction between vortices was found to be almost unaffected by the beta effect. Imposing a background flow with linear zonal shear on a pair of cyclonic vortices led to modification of the rotation rate of the vortices.

In the present work we continue to study the interaction of two tropical cyclones using a three-dimensional coupled atmosphere-ocean model. This study is different from Chang's (1983) work in three ways. First, the intensity and structure of the cyclones in a pair can freely change according to specified environmental conditions. Second, the evolution and motions of cyclones are considered on the beta plane. Third, the interaction with the ocean is included. It is a well-established fact that the sea surface temperature (SST) is a crucial parameter for a tropical cyclone development. Numerical models indicate a great sensitivity of the storm intensity to the SST, especially near the storm core (e.g., Chang 1979; Khain 1980; Tuleya and Kurihara 1982; Emanuel 1989). Numerous observational and numerical studies show rather strong sea surface cooling caused by rapid entrainment of cool thermocline water into the oceanic mixed layer induced by an intense wind stress under tropical cyclones. A typical SST decrease is found to range from $1^{\circ}$ to $6^{\circ} \mathrm{C}$ in response to hurricanes (Leipper 1967; Black 1983; Shay et al. 1992) and typhoons (Ramage 1974; Ivanov and Pudov 1977). The reduction of evaporation caused by the SST decrease may lead to substantial weakening of a TC. Recent numerical studies (Khain and Ginis 1991; Bender et al. 1993; Falkovich et al. 1993a,b) indicate that intensity, structure, and even tracks of tropical cyclones may be significantly modified by the interaction with the ocean. Ocean coupling is expected to be even more pronounced during binary tropical cyclone interaction since in this case one cyclone might move over the cold wake produced by another cyclone.

In order to keep sufficiently fine resolution within the cyclone core regions and also to trace the storm evolutions for longer period of time (4 days), a movable nested five-grid system is designed in this study. The main objective of the present paper is to report the results of a numerical investigation of the binary cyclones-ocean interaction using the coupled tropical cyclone-ocean model. The primary questions addressed to this study are the following.

- What are the major features of storm development during the interaction of two tropical cyclones?

- Which conditions control the attraction (or repulsion) of the tropical cyclones in a pair?

- What is the role of the tropical cyclone-ocean interaction in the processes mentioned above?

The paper has a following structure. The description of the coupled model is given in section 2 . The numerical framework of the coupled model is presented in section 3. The results of numerical experiments are considered in section 4 . We discuss the obtained solutions in section 5 . The conclusions and summary can be found in section 6 .

\section{The coupled tropical cyclone-ocean model}

The coupled tropical cyclone-ocean model used in this study consists of an eight-level atmospheric model and a three-layer ocean model (Fig. 2). In this section we will briefly describe the main features of the atmospheric and oceanic models.

\section{a. The tropical cyclone model}

The atmospheric model is based on the primitive equation system in sigma coordinates on the beta plane given as (Falkovich 1988)

$$
\begin{gathered}
\frac{d u}{d t}-f v+\frac{\partial \Phi}{\partial x}+C_{p} \theta \frac{\partial\left(\zeta^{\lambda}\right)}{\partial x}=F_{u}^{h}+F_{u}^{\sigma}, \\
\frac{d v}{d t}+f u+\frac{\partial \Phi}{\partial y}+C_{p} \theta \frac{\partial\left(\zeta^{\lambda}\right)}{\partial y}=F_{v}^{h}+F_{v}^{\sigma}, \\
\frac{d \chi}{d t}+\frac{\partial(u \chi)}{\partial x}+\frac{\partial(v \chi)}{\partial y}+\frac{\partial(S \chi)}{\partial \sigma}=0, \\
\frac{d \theta}{d t}=Q+F_{\theta}^{h}+F_{\theta}^{\sigma},
\end{gathered}
$$




$$
\begin{gathered}
\frac{d q}{d t}=-C+F_{q}^{h}+F_{q}^{\sigma}, \\
\sigma \frac{\partial \Phi}{\partial \sigma}=-R T_{\nu} \chi,
\end{gathered}
$$

where $\zeta=p / 1000, \sigma=\left(\zeta-\zeta_{h}\right) / \chi, \chi=\zeta_{s}-\zeta_{h}, \lambda$ $=R / C_{p}, \theta=T \zeta^{-\lambda}, T_{v}=T(1+0.61 q)$, and $d / d t$ $=\partial / \partial t+u \partial / \partial x+v \partial / \partial y+S \partial / \partial \sigma$.

Here $u, v$ are the components of the velocity vector in $x$ and $y$ direction, respectively; $S$ is the vertical velocity in sigma coordinates; $\theta$ is potential temperature; $\Phi$ is geopotential; $q$ is the water vapor-air mixing ratio; $P$ is pressure; $f$ is the Coriolis parameter at the beta plane; $R$ is the dry-air gas constant; $C_{p}$ is the specific heat at constant pressure; $F_{i}^{h}$ and $F_{i}^{\sigma}$ are the horizontal and vertical turbulent fluxes of the $i$ th variable $(i=u$, $v, \theta, q)$; and $Q$ and $C$ are the rates of latent heat release and condensation, respectively.

The term $\zeta$ is nondimensional pressure with the values of $\zeta_{s}$ and $\zeta_{h}$ at the lower and upper levels, respectively. Here $\chi$ and $S$ are calculated as follows:

$$
\begin{gathered}
\frac{\partial \chi}{\partial t}=-\int_{0}^{1}\left[\frac{\partial(u \chi)}{\partial x}+\frac{\partial(v \chi)}{\partial y}\right] d \sigma, \\
S=\frac{1}{\chi}\left\{\sigma \int_{0}^{1}\left[\frac{\partial(u \chi)}{\partial x}+\frac{\partial(v \chi)}{\partial y}\right] d \sigma\right. \\
\left.-\int_{0}^{\sigma}\left[\frac{\partial(u \chi)}{\partial x}+\frac{\partial(v \chi)}{\partial y}\right] d \sigma\right\} .
\end{gathered}
$$

The vertical structure of the model consists of eight levels in the troposphere. Above the troposphere, an isentropic layer placed as first suggested by Shuman and Hovermale (1968). The isentropic layer is given by the shallow-water equations written as follows:

$$
\begin{gathered}
\frac{\partial u_{i}}{\partial t}+u_{i} \frac{\partial u_{i}}{\partial x}+v_{i} \frac{\partial u_{i}}{\partial y}-f v_{i}+\frac{\partial \Phi_{i}}{\partial x}+C_{p} \bar{\theta} \frac{\partial\left(\zeta^{\lambda}\right)}{\partial x}=0 \\
\frac{\partial v_{i}}{\partial t}+u_{i} \frac{\partial v_{i}}{\partial x}+v_{i} \frac{\partial v_{i}}{\partial y}+f u_{i}+\frac{\partial \Phi_{i}}{\partial y}+C_{p} \bar{\theta} \frac{\partial\left(\zeta^{\lambda}\right)}{\partial y}=0 \\
\frac{\partial \zeta_{h}}{\partial t}+u_{i} \frac{\partial \zeta_{h}}{\partial x}+v_{i} \frac{\partial \zeta_{h}}{\partial y}+\zeta_{h}\left(\frac{\partial u_{i}}{\partial x}+\frac{\partial v_{i}}{\partial y}\right)=0 .
\end{gathered}
$$

Here $u_{i}$ and $v_{i}$ are the components of the horizontal velocity, $\bar{\theta}$ is the potential temperature of the layer assumed to be constant and $10 \mathrm{~K}$ larger than the initial value of potential temperature at the upper model level in the troposphere. Thus, the upper part of the atmosphere is always very stable. The results of supplemental experiments indicate that the isentropic layer helps to reduce the vertical velocity near the upper boundary and hence to decrease substantially the computational noise. Condensation heating is calculated at resolvable grid scales (Rosenthal 1978; Khain 1979, 1988) so that convection is hydrostatic but explicit. Horizontal turbulent fluxes are parameterized using a nonlinear viscosity scheme similar to that of Kurihara and Tuleya (1974). The vertical turbulent coefficient is assumed to be proportional to the vertical wind shear and is calculated as in Khain (1979). All variables at the anemometer level and the fluxes of sensible and latent heat and momentum are calculated using the Deardorff (1972) parameterization.

\section{b. The ocean model}

The ocean model is a reduced gravity, stratified model that is described in detail in Khain and Ginis (1991). It is based on momentum, heat balance, and continuity equations written in the Boussinesq and quasi-static approximations on the beta plane. The vertical structure of the model efficiently represents an important feature of the upper ocean involved in the response to TC forcing: the surface mixed layer and the seasonal thermocline. The surface mixed layer is a turbulent boundary that exchanges momentum and heat with the atmosphere at its surface and with the thermocline at the base. Normally the mixed layer is well mixed and, therefore, vertically uniform in temperature. The second layer (seasonal thermocline) is continuously stratified. The shape of the vertical temperature profile is given in the form of a nondimensional function that can be arbitrarily specified. For the calculations discussed in the present study, the temperature profile in the thermocline is specified in the form of the parabolic function. The interfacial (transition) layer with a sharp change of temperature at the base of the mixed layer is modeled by a discontinuity surface. The third layer is an abyssal layer of infinite depth and is assumed to be motionless and uniform in temperature. This is a common simplification for a reduced gravity model that is appropriate for studies of ocean response to a $\mathrm{TC}$ in the open ocean far from the coastlines. The horizontal velocity vector is assumed to be vertically uniform within each layer. The vertical turbulent mixing is the only subgrid-scale process treated in the model. As it has been shown in the previous studies (e.g., Price 1981; Ginis and Dikinov 1989), this process largely contributes to the ocean response to a hurricane forcing and, therefore, must be accurately parameterized. Three mechanisms of the turbulent kinetic energy production in the mixed layer are usually invoked: near-surface wind-driven shear, velocity shear at the base of the mixed layer, and convection due to the surface buoyancy fluxes. In a layered model the turbulent mixing is represented by the rate of entrainment of the thermocline water into the mixed layer. In the present work we use a multilimit entrainment model, developed by Deardorff (1983), that utilizes combined forcing effects of the mixed-layer turbulent production. 


\section{Numerical design}

\section{a. Mesh structure and numerical method}

The configuration of mesh nesting in the atmosphere model consists of five meshes (Fig. 3). The outermost mesh has $3840 \mathrm{~km} \times 3840 \mathrm{~km}$ size and $160-\mathrm{km}$ grid spacing. Each tropical cyclone in the pair has two telescopically nested meshes of $1600 \mathrm{~km} \times 1600 \mathrm{~km}$ and $800 \mathrm{~km} \times 800 \mathrm{~km}$ sizes with grid spacings of 80 and $40 \mathrm{~km}$, correspondingly. The specification of the boundary conditions at the interfaces between the meshes is based on the radiation method originally proposed by Orlanski (1976) and modified by Raymond and Kuo (1984) for multidimensional flows. The radiation boundary condition utilized in the present study is a two-dimensional type adjusted by Falkovich and Kulakov (1986) and Falkovich (1986) to the LaxWendroff time-integration scheme. It should be noted that the fundamental requirements for application of the radiation method, as pointed out by Pekelis and Pressman (1982), are that 1) one wave should predominate in some time-space vicinity of every grid point near the boundary; 2 ) the phase velocity of this wave should be a slowly changing function of time and space; and 3 ) the phase and group velocities need to have the same sign in the vicinity of the boundary. In order to satisfy these conditions in the present numerical scheme, the difference of the solutions in the two adjacent meshes of fine and coarse resolutions is radiated through the mesh interface. Specially designed test experiments (Falkovich 1986) indicate that this method allows long gravity waves to propagate through the boundaries between the computational domains of different resolutions with very small distortions.

The nested-mesh system considered in this study belongs to a class of two-way nesting systems. That is, the time integration proceeds simultaneously for fineand coarse-resolution meshes so that the adjacent domains interact dynamically with each other. The numerical scheme is designed in such a way that the meshes of different cyclones can cross each other. In this case, the boundary conditions are calculated only at the external boundaries of both meshes. For each grid point in the area common for two overlapping domains, the meteorological variables are assumed to belong to that tropical cyclone whose center is located closer to the grid point. The outermost mesh is motionless, while the internal nested meshes move with the corresponding cyclone. The movement of internal nests is designed as follows: when the storm moves by two space increments of the innermost mesh in any direction, it is displaced by one space increment of the middle mesh in the same direction. When the innermost mesh moves by two space increments of the middle mesh, the middle mesh is displaced by one space increment of the outermost mesh. After each displacement, the values of meteorological variables at the two grid rows nearest to the boundary in the direction of the movement are

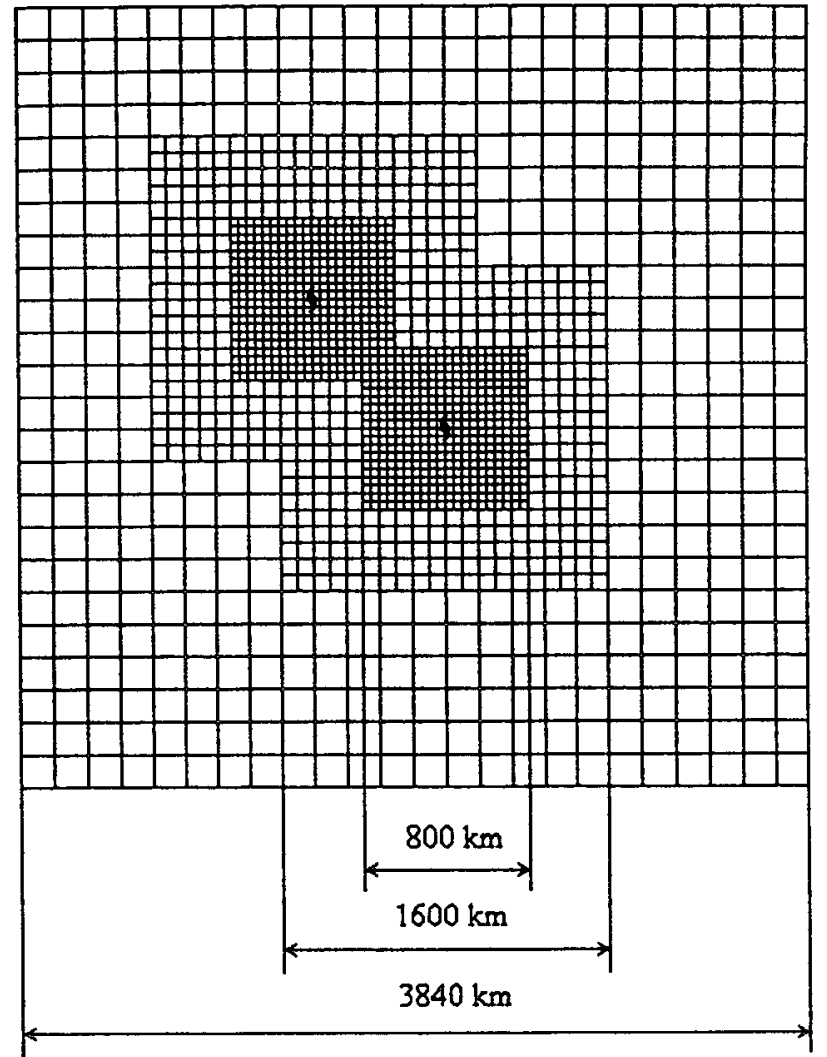

Fig. 3. The mesh configuration in the tropical cyclone model.

obtained by linear interpolation of the values at the coarse grids.

The time integration is performed with different time increments for each integration domain that are equal to 6,3 , and $1.5 \mathrm{~min}$, respectively, for the outermost, middle, and innermost domains. The rules defining the order of time integration are similar to the ones used by Kurihara et al. (1979). A version of the Lax-Wendroff scheme proposed by Pressman (1968) is applied for the time integration at each time step. In this scheme the staggering is used both in horizontal and vertical coordinates.

The oceanic domain contains $107 \times 107$ grid points with the grid spacing of $40 \mathrm{~km}$. The finite-difference equations for the ocean model are formulated on the staggered Arakawa B grid. The time integration is performed based on a splitting method developed by Marchuk (1982) with the time increment of $30 \mathrm{~min}$. Ginis and Sutyrin (1995) demonstrate how the splitting method can be effectively applied for the integration of the layered model equations.

\section{b. Initial conditions and the sequence of computations}

In all numerical experiments discussed in this paper, a pair of equally strong axisymmetric vortices of the 
Fiorino and Elsberry (1989) type is placed at varies separation distances $d$. The radius of the maximum winds $R$ is equal to $120 \mathrm{~km}$. Initial vertical distribution of the maximum tangential velocity is specified as $v_{m}$ $=15 \mathrm{~m} \mathrm{~s}^{-1} \times(1-z / 16600)$, where $z$ is the height in meters. The highest model level is initially located near $100 \mathrm{mb}$, which corresponds to $\zeta_{h}=0.1$. The Jordan (1958) mean vertical temperature profile typical of the tropical atmosphere in a hurricane season is used initially. Relative humidity in the lower troposphere is set to be $95 \%$, and the SST is set to be $301 \mathrm{~K}$.

Note that according to Emanuel's (1991) theory, the maximum potential intensity of TC (the potential minimum sea surface pressure) can be evaluated as a little less than $900 \mathrm{mb}$.

The ocean is initially horizontally homogeneous and quiescent. The depths of the mixed layer and the thermocline are equal to 30 and $500 \mathrm{~m}$, respectively. The temperatures at the top and bottom of the thermocline are $26.5^{\circ}$ and $10^{\circ} \mathrm{C}$.

The order of computations of the coupled model is as follows: during the period of one time step in the ocean model, the TC model is integrated using its own time steps for different meshes while the SST remains unchanged. The net heat flux (sensible and latent fluxes) and the wind stress calculated in the atmospheric model are then passed into the ocean model for the next sequential step to modify the thermodynamic structure of the ocean. New SST values, obtained as a result of ocean mixing, upwelling, and evaporation from the sea surface, are used in the subsequent integration of the TC model. In this way the ocean influences the TCs through the heat and moisture fluxes at its surface.

\section{Results of numerical experiments}

As indicated before, the purpose of this study is to investigate the evolution and motion of binary tropical cyclones and the possible influence of the ocean coupling on the behavior of the pair of storms. To accomplish this task we conducted a series of numerical experiments (hereafter referred to as $\mathrm{AO}$ ) with the coupled atmosphere-ocean model described in the preceding sections. In an attempt to isolate the oceanic effects, we additionally performed simulation experiments in which SST was fixed to a constant value (these experiments are hereafter referred to as A). The comparison of the coupled and uncoupled experiments helped to determine how much of the alteration of the storm structure and movement in the pair was due to the effect of ocean interaction.

In all of the experiments discussed below, two identical vortices were initially separated by a specified distance (Table 1). The vortices were initially located at $20^{\circ} \mathrm{N}$ for most of experiments. In two model integrations, that is experiments $\mathrm{A} 2$ and $\mathrm{AO} 2$, binary vortices were initially located in the northeast-southwest direc-
TABLE 1. Summary of the numerical experiments.

\begin{tabular}{|c|c|c|c|c|}
\hline \multirow[b]{2}{*}{ Experiment } & \multicolumn{2}{|c|}{$\begin{array}{c}\text { Separation } \\
\text { distance }(\mathbf{k m})\end{array}$} & \multirow{2}{*}{$\begin{array}{l}\text { Minimum } \\
\text { surface } \\
\text { pressure (mb) }\end{array}$} & \multirow{2}{*}{$\begin{array}{l}\text { Time of } \\
\text { merging }\end{array}$} \\
\hline & $t=0$ & $t=96 \mathrm{~h}$ & & \\
\hline AF & 320 & 528 & $\begin{array}{l}\text { W } 906 \\
\text { E } 908\end{array}$ & - \\
\hline A1 & 320 & 500 & $\begin{array}{l}\text { W } 907 \\
\text { E } 946\end{array}$ & - \\
\hline AOI & 320 & 524 & $\begin{array}{l}\text { W } 940 \\
\text { E } 945\end{array}$ & - \\
\hline $\mathrm{A} 2$ & 450 & 528 & $\begin{array}{l}\text { W } 908 \\
\text { E } 911\end{array}$ & - \\
\hline $\mathrm{AO} 2$ & 450 & 740 & $\begin{array}{l}\text { W } 955 \\
\text { E } 938\end{array}$ & 一 \\
\hline A3 & 280 & 0 & $\begin{array}{l}\text { W } 928 \\
\text { E } 952\end{array}$ & $t=79 \mathrm{~h}$ \\
\hline $\mathrm{AO} 3$ & 280 & 592 & $\begin{array}{l}\text { W } 943 \\
\text { E } 949\end{array}$ & - \\
\hline A4 & 240 & 0 & $\begin{array}{l}\text { W } 958 \\
\text { E } 960\end{array}$ & $t=31 \mathrm{~h}$ \\
\hline $\mathrm{AO} 4$ & 240 & 0 & $\begin{array}{l}\text { W } 965 \\
\text { E } 965\end{array}$ & $t=31 \mathrm{~h}$ \\
\hline
\end{tabular}

tion with the midpoint located at $20^{\circ} \mathrm{N}$. As seen in Table 1 , the initial distances between the storms are rather short (about $300 \mathrm{~km}$ ) compared with the average separation distance of $1000 \mathrm{~km}$ observed by Lander and Holland (1993). However, they are longer than the minimum separation distance of $180 \mathrm{~km}$ found by Dong and Neumann (1983). The initial distances in Table 1 also correspond well to the numerical studies of Ritchie and Holland (1993) and Holland and Dietachmayer (1993), who found that the behavior of binary storms was mutually depended when the separation distances were about $300 \mathrm{~km}$. In order to compare the evolution of the storm pair on the $f$ and beta planes, a constant Coriolis parameter was used in one of the experiments, which we call experiment AF. In all other cases presented here the beta-plane approximation is assumed. In this section we will discuss some of the interesting features related to the storm tracks and structure revealed in the experiments performed. In the following discussion, for the sake of convenience, we shall refer to the vortex initially located to the west (or east) by the letter W (or E).

\section{a. Experiment on the f plane}

We begin the discussion with noninteractive experiment $\mathrm{AF}$ in which the Coriolis parameter was set as a constant. The storm tracks for this experiment are shown in Fig. 4. Since each storm moved only in the steering flow induced by the circulation of the other one, the storms moved cyclonically around their intermediate point. The translational speeds of the storm increased for about $60 \mathrm{~h}$. This is apparently related to the fact that both storms undergo a rapid deepening due to very favorable environmental conditions. The trop- 


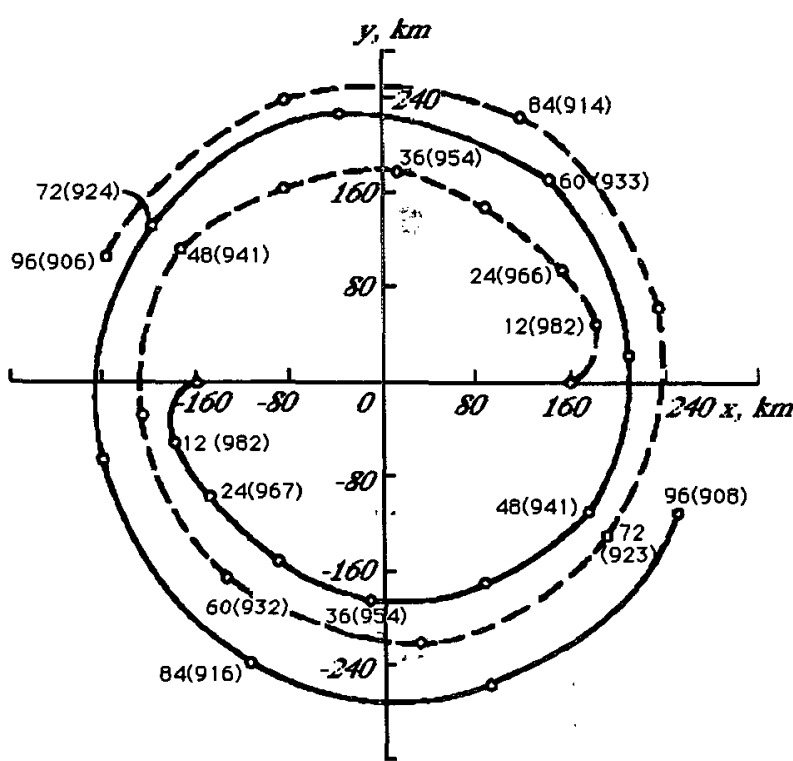

FIG. 4. The tracks of binary tropical cyclones in experiment AF. The storm $\mathrm{W}$ is shown by the solid line, storm $\mathrm{E}$ is shown by the dashed line. Numbers on the curves denote time (h) and minimum surface pressure (mb).

ical cyclones in this experiment reveal nearly symmetrical evolution. The time series of minimum sea surface pressure for storm $W$ is illustrated in Fig. 5. After an adaptation period of about $6 \mathrm{~h}$, storm $\mathrm{W}$ began to quickly intensify and reached supertyphoon strength of $908 \mathrm{mb}$ by $96 \mathrm{~h}$. It should be pointed out that the structure of the tropical cyclones in all experiments discussed in the present paper corresponds fairly well to the structure of real tropical cyclones. It is characterized by an inflow layer in the low troposphere, an outflow layer in the upper troposphere, and a warm core surrounded by an eyewall with intense updraft.

As seen in Fig. 4, the two tropical cyclones were moving slowly away from each other. The separation distance between the storm centers increased from 320 $\mathrm{km}$ at $t=0$ to $528 \mathrm{~km}$ at $t=96 \mathrm{~h}$. It is interesting to note that after about $60 \mathrm{~h}$ of integration, the translational speed of both storms remained almost the same until the end of integration despite the cyclones' continued deepening (Fig. 5). This is probably related to the fact that for the larger separation distances, the binary storms found themselves in weaker steering flows due to the decrease of the tangential winds with radius. The nearly constant translational speed of the storms after $60 \mathrm{~h}$ in this experiment indicates that the decrease of the magnitude of the tangential winds with radius was compensated by its increase due to the storm deepening.

\section{b. Experiments $A l$ and $A O I$}

The first experiment, A1, differs from the noninteracting experiment $\mathrm{AF}$ by only the variability of the Cor-

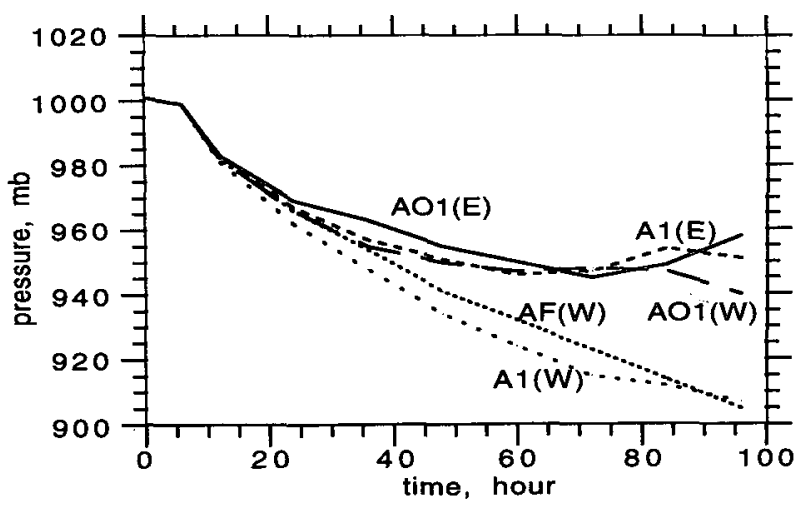

FIG. 5. Time series of the minimum surface pressure for the coupled (AO1) and noncoupled (AF, A1) experiments. Parameters of the experiments are presented in Table 1.

iolis parameter. That is, in addition to mutual rotation, the binary storms move northwest due to the beta effect. The storm tracks in experiment Al are shown in Fig. 6. During the early period of integration, the vortex $\mathrm{W}$ driven by the circulation of the vortex $\mathrm{E}$ was moving south and then, during the 12-40-h period, southeast. At the same time, the vortex $\mathrm{E}$, driven by the circulation of the vortex W, moved north and then northwest. Hence, the direction of storm $W$ movement at that period of time was against the beta drift, while the direction of storm $\mathrm{E}$ movement was in the direction of the beta drift. For this reason the vortex $\mathrm{W}$ was moving slower than the vortex $\mathrm{E}$. The average translational speeds of the storms during the 12-36-h period were 2.3 and $4.2 \mathrm{~m} \mathrm{~s}^{-1}$ for storms $\mathrm{W}$ and $\mathrm{E}$, respectively. The important consequence of this asymmetry in the

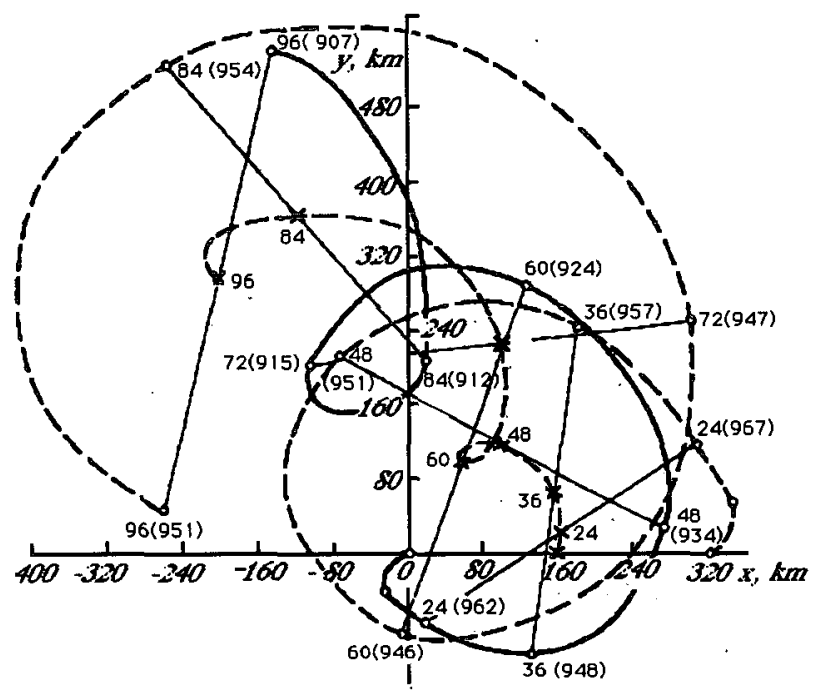

Fig. 6. The same as in Fig. 4 but for experiment A1. The dashed line with crosses shows the trajectory of the middle point between the centers of vortices. 


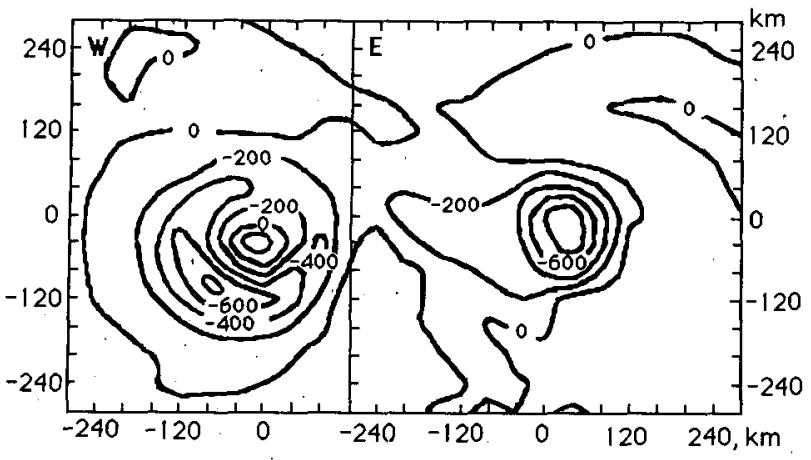

FIG. 7. Distribution of $\omega=d p / d t\left(\mathrm{mb} \mathrm{h}^{-1}\right)$ at level $6(\sim 550 \mathrm{mb})$ in experiment Al at $t=72 \mathrm{~h}$. The vortex located to the west at $t$ $=0$ is denoted by the letter $\mathrm{W}$, the other vortex is denoted by the letter $\mathrm{E}$.

storm movements is the difference in the evolution of the cyclones. One can see in Fig. 5 that storm $W$ is substantially deeper than storm E. We speculate that two possible mechanisms could contribute to the observed difference in the storm deepening rates. The first one is probably directly related to the difference in the storm translational speeds. Our speculation is based on the results of previous numerical studies (e.g., Bender et al. 1993; Falkovich et al. 1995) that show that more intense basic flows generally decrease the rate of storm intensification (may be due to increase of ventilation effects in the boundary layer). In order to deepen, a tropical storm needs to develop a warm core, to form an eye, etc. The more intense basic flow tends to impede these processes due to faster convection area replacement and boundary layer effects. The second mechanism is related to the fact that each storm in the pair moves in a steering flow with considerable vertical shear. The faster deepening rate of storm $\mathrm{W}$ during the initial period of integration resulted in the development of the stronger vertical shear in its circulation, which, in turn, further reduced the deepening of storm $E$. The tendency of a tropical cyclone to decrease its intensity with an increase of vertical shear in the environmental flows was noted in many studies (e.g., Gray 1979; Kurihara and Tuleya 1981; Shapiro 1992). The apparent increase in time of the asymmetries in storm intensities (up to $44 \mathrm{mb}$ at $96 \mathrm{~h}$ ) in experiment $\mathrm{A} 1$ is probably caused by the progressive increase of the difference in storm translation speeds and vertical shear structures.

Remarkable difference is also found in the storm structures. Figure 7 shows the vertical velocity distributions for cyclones $\mathrm{W}$ and $\mathrm{E}$ at $72 \mathrm{~h}$. Only inner parts of fine meshes are shown. At $72 \mathrm{~h}$ the cyclones are occasionally located in the west-east direction, as they were at $t=0 \mathrm{~h}$. At this time we see that storm W has a distinct area of downward vertical motion (the eye), whereas storm $\mathrm{E}$ has no developed eye. Note that the latter storm even started to weaken after 72 h (Fig. 6).
Another feature that can be noted is that storm $E$ is significantly weaker than either of the storms in experiment AF (Fig. 5). This again correlates well with the storm translational speeds in these experiments since the storms in experiment AF were moving slower than storm $\mathrm{E}$ in experiment $\mathrm{Al}$.

Experiment AO1 differs from experiment A1 by only the addition of ocean coupling. As a result of airsea interaction, the storms' evolution and motion were significantly different from those in experiment A1 (Figs. 5 and 8 ). The strong wind stress associated with the storms produced intense turbulent mixing in the upper ocean, resulting in significant SST decrease. Figure 9 shows the distribution of the SST anomalies at $72 \mathrm{~h}$. It is evident that the maximum sea surface cooling of about $3.5^{\circ} \mathrm{C}$ occurred in the areas where storms tracks were close or crossed each other. As a result of the SST decrease, both storms deepened more slowly (Fig. 5). Such behavior is in agreement with previous studies (Khain and Ginis 1991; Bender et al. 1993; Falkovich et al. 1993, 1995) that also demonstrated a reduction of storm intensity due to interaction with the ocean. The main mechanism responsible for the storm weakening is related to the decrease of the supply of latent heat energy to the storm caused by the decrease of the evaporation rate. Figure 5 indicates that the impact of ocean coupling was the greatest on storm W. The difference in minimum sea level pressure between this and the uncoupled experiment $\mathrm{Al}$ reached about $34 \mathrm{mb}$ at $96 \mathrm{~h}$. During the first $24 \mathrm{~h}$ as storm W was undergoing rapid strengthening, the effect on storm intensification was rather small. However, after $24 \mathrm{~h}$ without ocean interaction, the storm continued to rap-

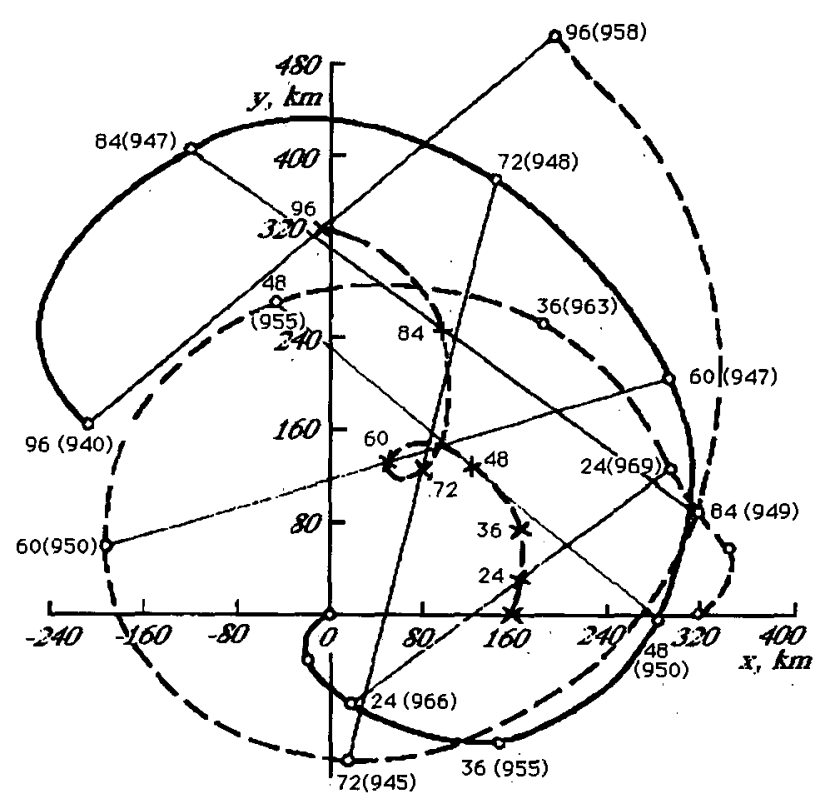

FIG. 8. As in Fig. 6 but for experiment AO1. 


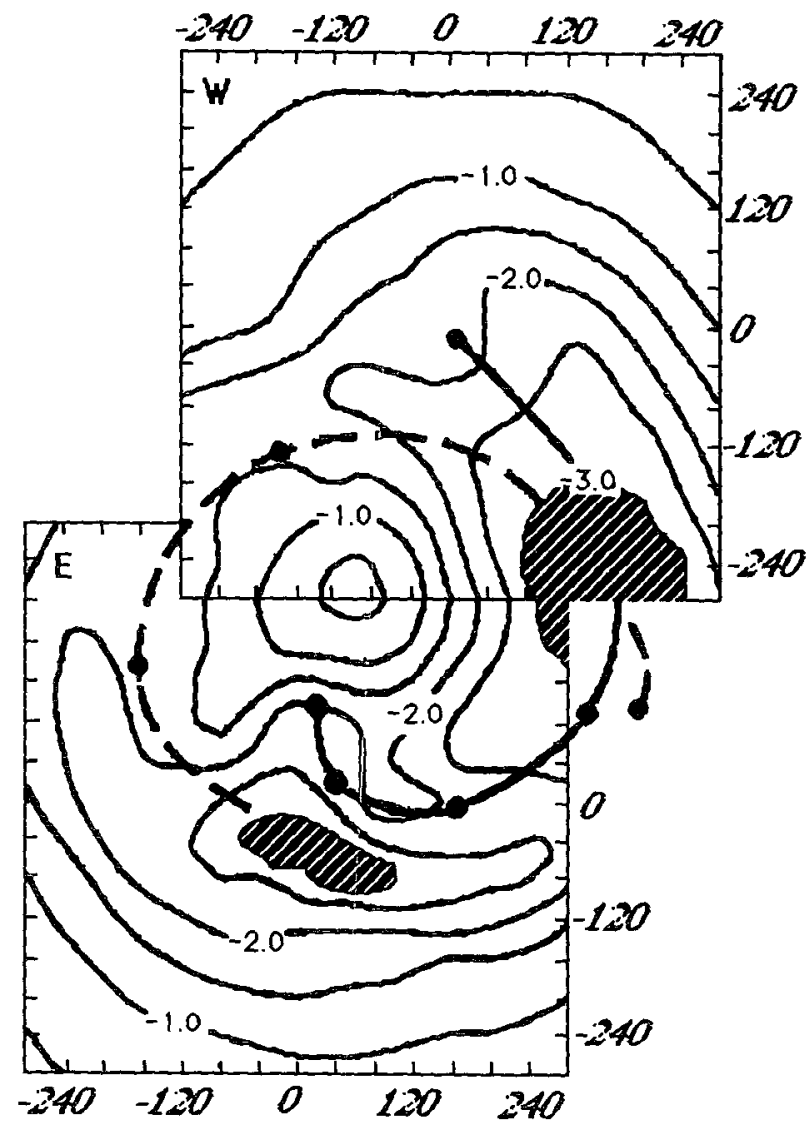

FIG. 9. Contours of the sea surface temperature anomalies $\left({ }^{\circ} \mathrm{C}\right)$ in experiment AOl at $t=72 \mathrm{~h}$.

idly intensify, whereas the interactive storm deepened considerably more slowly. The intensification rate of the latter storm even began to level off after $48 \mathrm{~h}$.

As we can see from Fig. 9 at that time storm W approached and then passed over the area with water already cooled by storm E. Hence, the strong weakening of storm $\mathrm{W}$ is mainly related to the fact that for a large period of time it moved over the wake of cold water generated by storm $\mathrm{E}$.

The evolution of storm $\mathrm{E}$ in experiment $\mathrm{AO} 1$ was quite different from storm W. For the first $72 \mathrm{~h}$ storm $\mathrm{E}$ was moving over the initially undisturbed ocean and, therefore, it had a cold wake only at the rear part of the storm produced by itself. Accordingly, the rate of the storm intensification was only moderately less than that in the uncoupled experiment. For example, the difference in the minimum sea level pressure from experiment $\mathrm{Al}$ at $48 \mathrm{~h}$ was only about $4 \mathrm{mb}$. However, after $72 \mathrm{~h}$, when storm $\mathrm{E}$ was passing over the cold water produced by storm W (Fig. 9) it began to weaken (Fig. 5 ), probably due to an additional decrease of latent heat flux in the frontal area of the storm. Hence, although storm $\mathrm{E}$ in both experiments $\mathrm{A} 1$ and $\mathrm{AO} 1$ was weakening during the period from 72 to $96 \mathrm{~h}$, the decrease was caused by different mechanisms (ocean interaction in experiment AOI and fast translation speed in experiment A1). Note that because of the different responses of the storms $\mathrm{E}$ and $\mathrm{W}$ to the ocean coupling, the final difference in minimum pressure in the experiment $\mathrm{AO} 1$ was about $18 \mathrm{mb}$, in contrast to experiment $\mathrm{Al}$ when this difference was $44 \mathrm{mb}$.

The ocean interaction also introduced a significant effect on the storm tracks (compare Figs. 6 and 8 ). Since the circulation of one storm is a basic flow for another one in a storm pair, the weakening of storm $W$ caused the slower rotation of storm $\mathrm{E}$. As a result, the tracks of both storms were changed dramatically. For instance, at $96 \mathrm{~h}$ storm $\mathrm{E}$ in experiment $\mathrm{AO} 1$ was located over $640 \mathrm{~km}$ to the northeast of the same storm in experiment A1. We can thus conclude that ocean coupling may greatly affect not only the tropical cyclone strengths but also their motions in the case of binary storm interaction. We finally note that similar to the other cases discussed so far, the separation distance between storms in experiment AO1 gradually has increased in the course of integration. No significant difference was found in comparison with experiment $\mathrm{A} 1$ (Table 1).

\section{c. Experiments $A 2$ and $A O 2$}

In this pair of experiments the binary storms were initially separated by a distance of $450 \mathrm{~km}$ in the southwest-northeast direction (Figs. 10 and 11). Because of the larger separation distance, the binary storms were rotating around each other with smaller speeds than those in experiments $\mathrm{Al}$ and $\mathrm{AO}$. This resulted in not only differences in the storms' tracks but also in their intensities and strengths. Figure 12 shows time series of the minimum sea level pressures for the storms W and $E$ in experiments $\mathrm{A} 2$ and $\mathrm{AO} 2$. We immediately notice that, in contrast to experiment $\mathrm{A} 1$, storm $\mathrm{E}$ in experiment A2 was deepening at only a slightly lower rate than storm $W$. Such behavior is apparently related to the reduction of the translational speed of storm $E$. Both storms, E and W, attained a supertyphoon strength by $96 \mathrm{~h}$.

The interaction with the ocean weakened both storms. However, the effect was larger on storm W. It was deepening substantially more slowly than the analogous storm in the noncoupled experiment. After about $60 \mathrm{~h}$ storm $\mathrm{W}$ began to weaken. Examination of the storm track (Fig. 11) indicates that during this time storm $\mathrm{W}$ approached the region previously occupied by storm $E$ so that it was moving close to the track (and consequently over the cold wake) of storm $\mathrm{E}$. It is interesting to note that because of the 1.5 times smaller transition velocity and, consequently, stronger coupling with the ocean, storm $\mathrm{W}$ weakened more rapidly in this experiment than it did in experiment AO1. For example, the minimum sea level pressure is $955 \mathrm{mb}$ at $96 \mathrm{~h}$ in experiment $\mathrm{AO} 2$, as compared with $940 \mathrm{mb}$ in experiment AO1. 
We also notice the profound effect of the ocean coupling on the repulsion process. The separation distance increased much more quickly in experiment $\mathrm{AO}_{2}$ in comparison with experiment A2. At $t=96 \mathrm{~h}$ the distance between the storms was $528 \mathrm{~km}$ in experiment A2, whereas it reached $740 \mathrm{~km}$ in experiment $\mathrm{AO} 2$. The probable explanation for this effect will be given in the next section where the mechanisms of attractionrepulsion will be discussed.

\section{d. Experiments $A 3$ and $A O 3$}

These experiments are of special interest because the binary storms merged in experiment $\mathrm{A} 3$, but they moved away in experiment $\mathrm{AO} 3$. The experiments are similar to experiments $\mathrm{A} 1$ and $\mathrm{AO} 1$ except that the initial separation distance between vortices was set to 280 $\mathrm{km}$. Even the small difference of $40 \mathrm{~km}$ in the separation distance caused substantial modification in the storm tracks and intensities (Figs. 13 and 14) in the noncoupled experiment. Both storms, $W$ and $E$, were weaker than the corresponding ones in experiment A1, probably due to faster mutual rotation. For example, at $72 \mathrm{~h}$ the minimum sea level pressure was $952 \mathrm{mb}$ for storm E and $928 \mathrm{mb}$ for storm W compared with 947 and $915 \mathrm{mb}$ for the corresponding storms in experiment A1. In experiment $A 3$ the storms merged at $t=79 \mathrm{~h}$. In order to analyze how the merger process occurs in the model, the instantaneous precipitation fields are

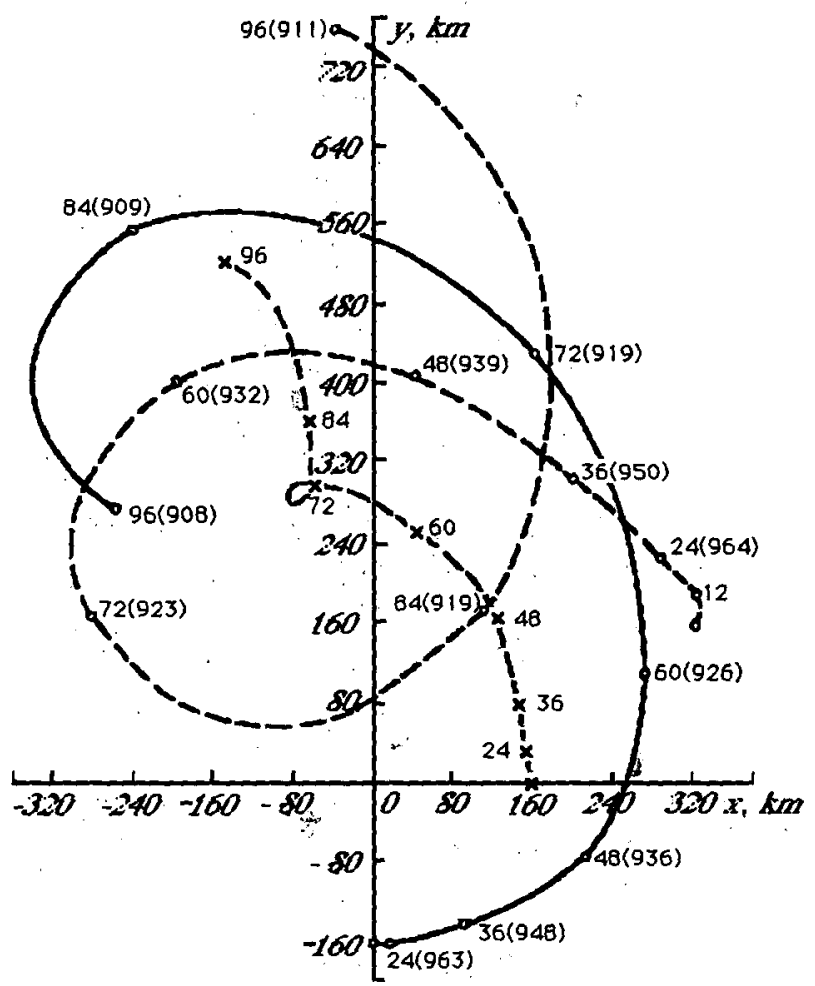

FIG. 10. As in Fig. 6 but for experiment A2.

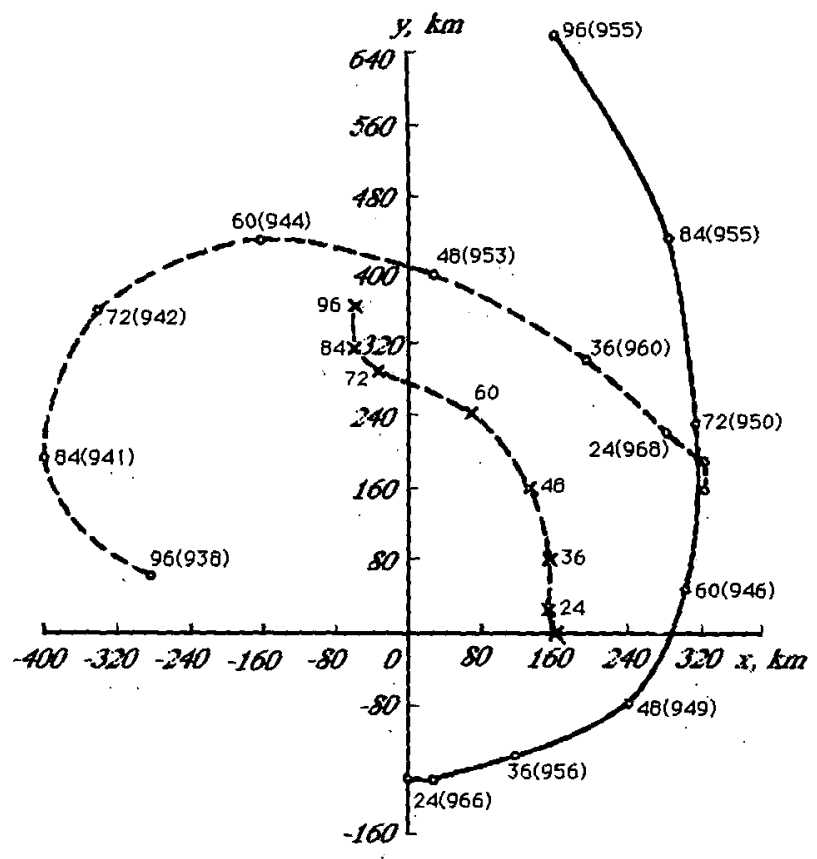

FIG. 11. As in Fig. 6 but for experiment AO2.

plotted at $48 \mathrm{~h}$ (Fig. 15a) and at $72 \mathrm{~h}$ (Fig. 15b). It is seen that at $48 \mathrm{~h}$ ( $31 \mathrm{~h}$ before merger) the two fields were quite well separated from each other by an area of almost no rainfall. Although the maximum precipitation rates for both storms were nearly the same (120$140 \mathrm{~mm} \mathrm{~h}^{-1}$ ), the area-integrated amount of precipitation was greater for the stronger vortex W (not shown). Over the 24-h period, from 48 to $72 \mathrm{~h}$, the precipitation maxima and the distance between these maxima did not change much. For example, the separation distance decreased only from 352 to $304 \mathrm{~km}$. However, the precipitation distribution was remarkably different at $72 \mathrm{~h}(7 \mathrm{~h}$ before merger) from the one at $48 \mathrm{~h}$. The precipitation pattern of the weaker storm $\mathrm{E}$

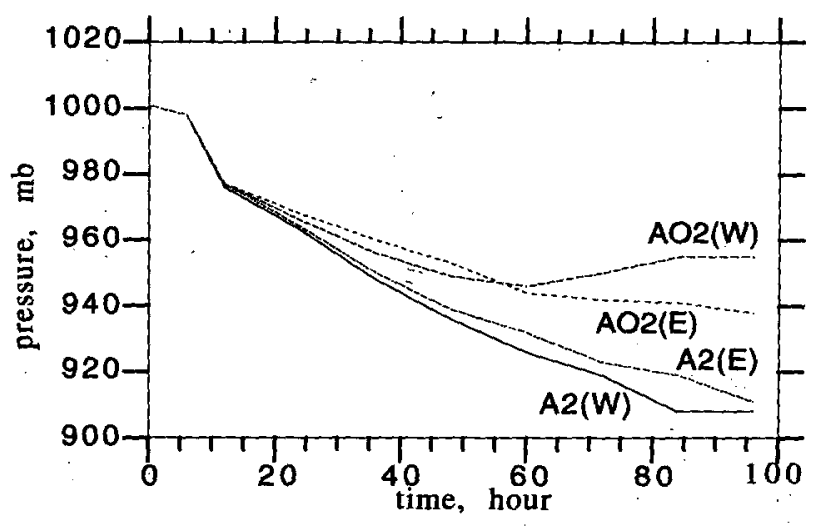

FIG. 12. Time series of the minimum surface pressure in experiments $\mathrm{A} 2$ and $\mathrm{AO} 2$. 


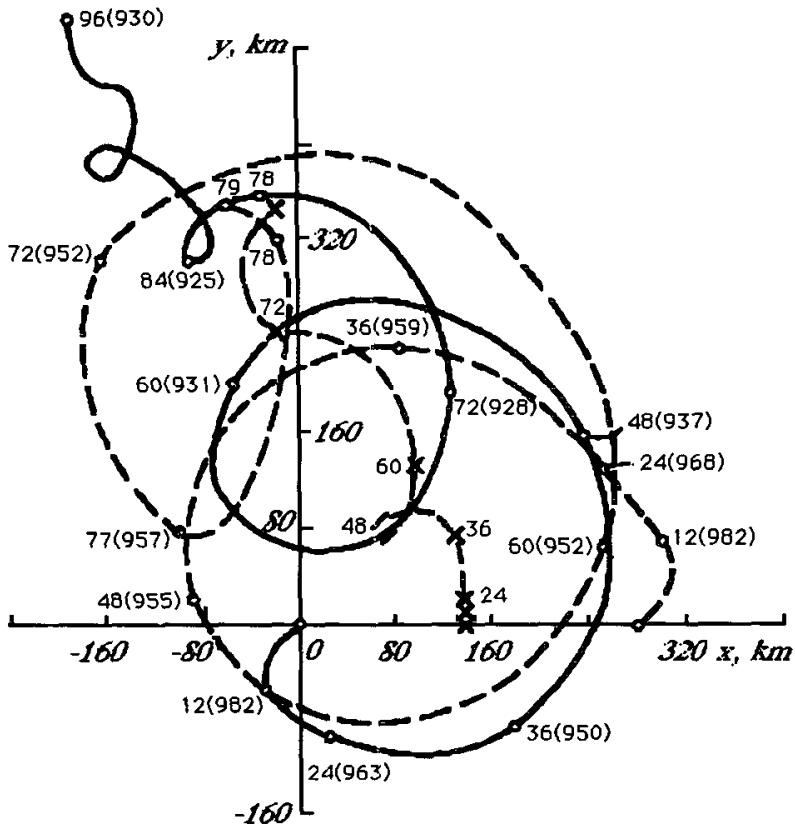

Fig. 13. The tracks of binary tropical cyclones in experiment A3. Designations are the same as in Fig. 4.

became extended toward the stronger storm $\mathrm{W}$. Thus, a part of the precipitation field of storm $E$ turned into a spiral band of storm $W$. Within the band, precipitation is clearly nonuniform and has several maxima. The main maximum of precipitation (along with the maxima of vertical velocity and relative vorticity) still belonged to storm $\mathrm{E}$ at that time. However, the peripheral maxima can already be attributed to the precipitation field (and vertical motion and vorticity fields) of storm W. Analysis of the precipitation patterns during the next $7 \mathrm{~h}$ preceding the storm merger indicated that the peripheral maxima grew with time, whereas the main maximum decreased. By $79 \mathrm{~h}$ storm $\mathrm{E}$ became inseparable from the storm $W$ in field of the surface pressure and the whole storm $E$ turned into a rainband of storm $W$. Thus, in our model the process of merger of binary tropical cyclones seems to be a displacement of maximum vorticity, vertical velocity, and corresponding thermodynamical fields of the weaker storm in the direction of the stronger storm along the spiral bands. This finding seems to be consistent with observed data (Lander and Holland 1993).

As a result of the interaction with the ocean, the binary storms in experiment AO3 moved away from each other (Fig. 16). It is interesting to compare the storms' behavior in this experiment with that in experiment A01. Similar to the noninteracting case discussed above, a small decrease of the initial separation distance caused significant impact on the tracks of the tropical cyclones (compare Figs. 16 and 8). Repulsion in this experiment occurred more rapidly than that in experiment AO1. Over the $96 \mathrm{~h}$ of integration, the sep-

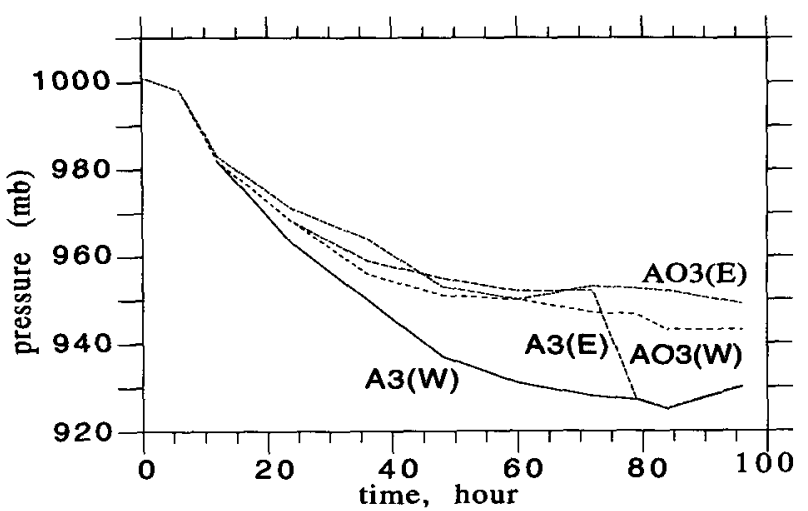

FIG. 14. Time series of the minimum surface pressure in experiments $\mathrm{A} 3$ and $\mathrm{AO} 3$.

aration distance increased from 280 to $592 \mathrm{~km}$, compared with the increase from 320 to $524 \mathrm{~km}$ in experiment AO1 (Table 1). However, these changes in the

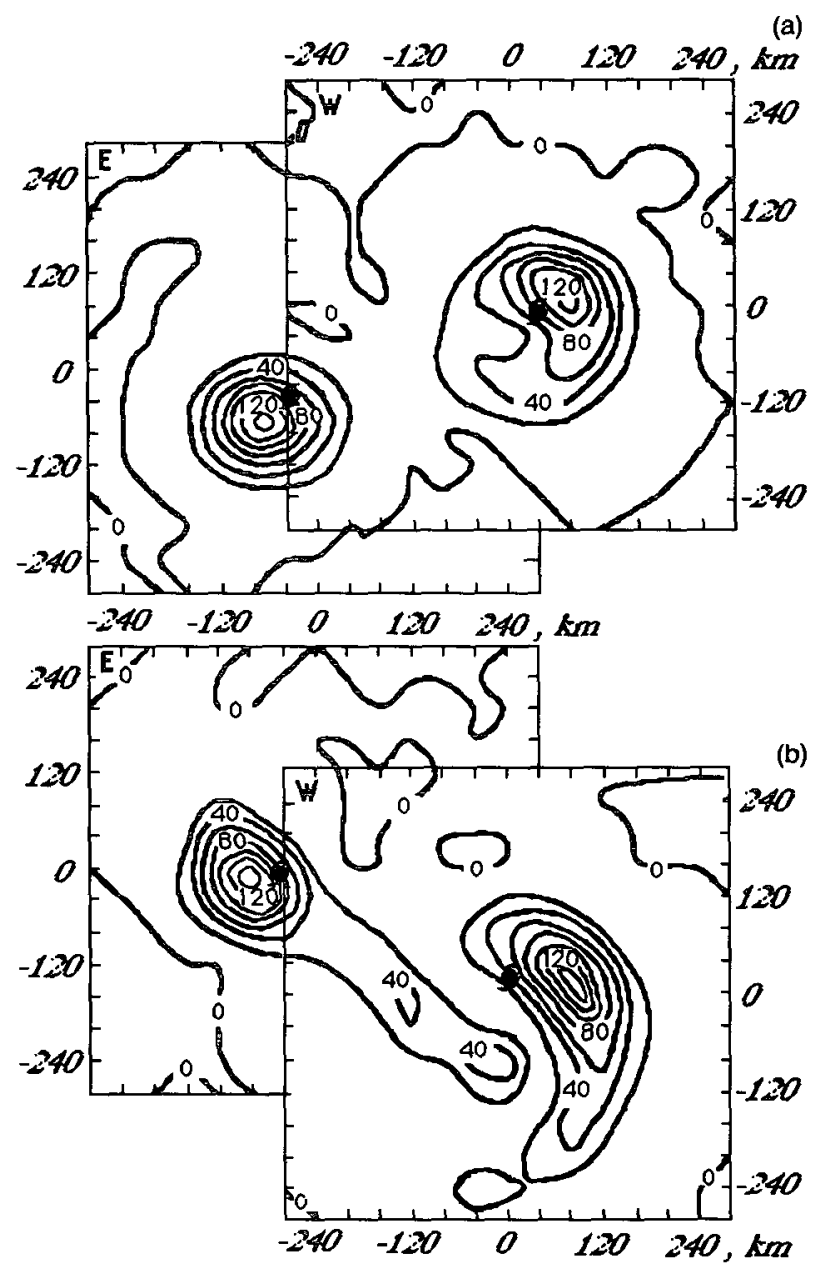

FIG. 15. Precipitation rate $\left(\mathrm{mm} \mathrm{h}^{-1}\right)$ in experiment $\mathrm{A} 3$ at (a) $t=48 \mathrm{~h}$ and (b) $t=72 \mathrm{~h}$. 


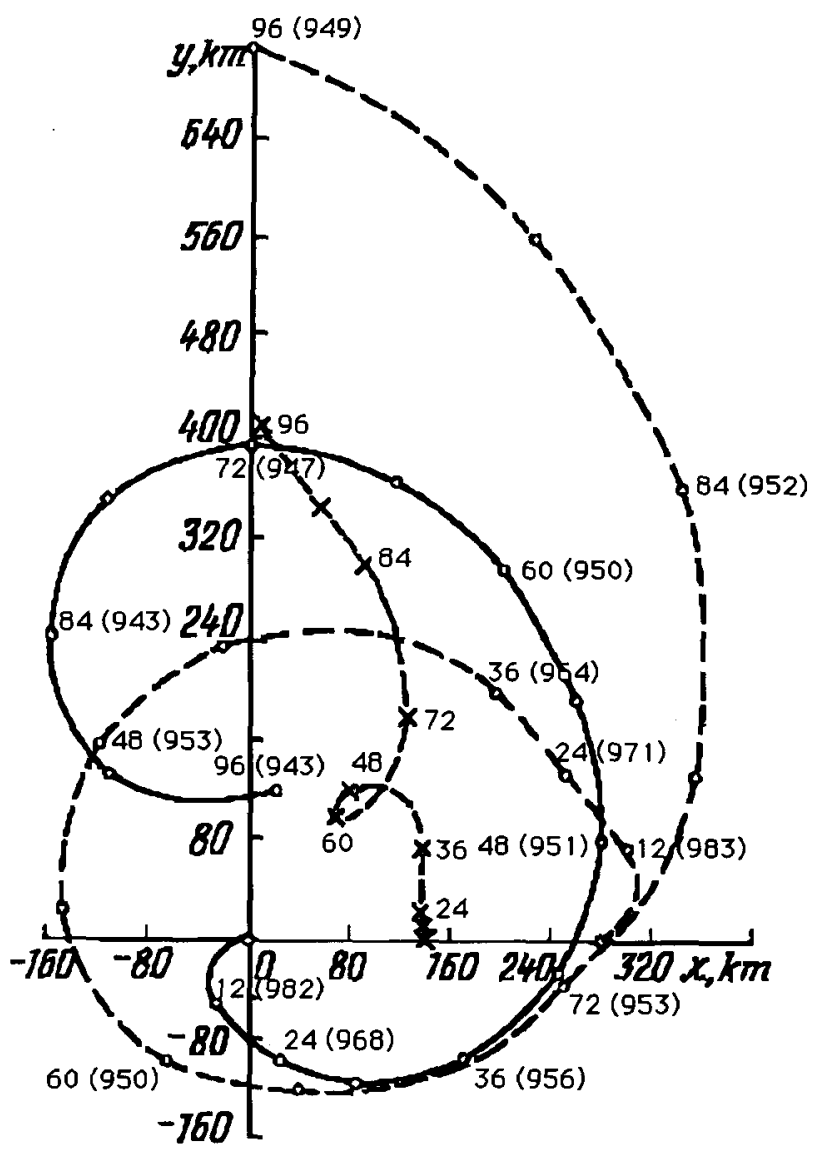

Fig. 16. As in Fig. 13 but for experiment AO3.

tracks have not greatly affected the storm intensities. By $96 \mathrm{~h}$ the minimum sea level pressures of both storms in experiment $\mathrm{AO} 3$ were very close to those in exper- iment AO1 (Table 1). Some differences can only be found in the time evolution of the storms (especially for storm E) (compare Figs. 14 and 5) that are apparently related to the different amounts of time spent by each vortex over the cold wake generated by the other one.

\section{e. Experiments $\mathrm{A} 4$ and $\mathrm{AO} 4$}

The pair of vortices was separated by a distance of $240 \mathrm{~km}$ in these experiments. This resulted in rapid attraction of the vortices and their merger at $31 \mathrm{~h}$ (Fig. 17). No apparent difference in the storms' behavior between experiments $\mathrm{A} 4$ and $\mathrm{AO} 4$ is found. This is probably because of high translation velocity of TCs in these experiments and, as a result, the weak coupling of the storm with the ocean in experiment $\mathrm{AO} 4$. The storms in experiment $\mathrm{AO} 4$ were rather too weak to produce a SST decrease sufficient to make a noticeable impact on the structure and intensity of the storms during a short time period before the merger $(t=30 \mathrm{~h})$. However, after the merger, the difference between the newly emerged storms in experiment A4 and AO4 became more apparent: at $48 \mathrm{~h}$ the minimum sea level pressure was $930 \mathrm{mb}$ in experiment A4, against $947 \mathrm{mb}$ in experiment $\mathrm{AO} 4$.

The small initial separation distance caused the storms in these experiments to move rather rapidly by their mutual advection. No apparent asymmetry developed in storm intensities and structures during the time before the merger.

As a result, the merger process in this case was quite different from that in experiment A3. Analysis indicated that during the merger the storms turned into two equally intense spiral bands of a newly emerged tropical cyclone. Thus, the way in which binary storms

(b)

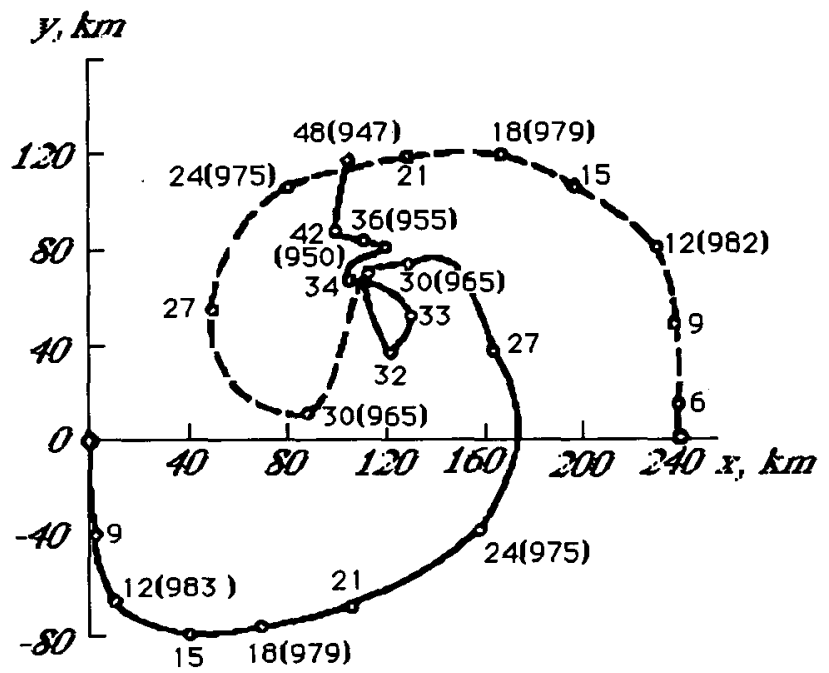

FIG. 17. The tracks of binary tropical cyclones in experiments (a) A4 and (b) AO4. Designations are the same as in Fig. 4. 
merge seems to depend on their relative intensities and strengths.

The difference in merger processes between binary vortices of either different or the same strengths was also noted by Pokhil (1991) in experiments with a barotropic model.

\section{Mechanisms influencing the merger of two vortices}

In this section we will discuss some possible mechanisms responsible for attraction-repulsion of binary tropical cyclones. In the experiments presented in the preceding section, merger occurred (did not occur) if the initial separation distance $d$ was less (more) than its critical value $d_{\mathrm{cr}}$. The experimentally found magnitude of $d_{\mathrm{cr}}$ is about $300 \mathrm{~km}$ for the noncoupled experiments and is about $260 \mathrm{~km}$ for the coupled experiments.

One of the possible effects that determines whether two interacting vortices will merge or separate depends on the vorticity distribution of the combined system (DeMaria and Chan 1984; Chan 1993). In order to analyze this effect, we plot in Figs. 18 and 19 the vorticity fields at the middle model level $(\sigma=0.5)$ for experiments A1 ( $72 \mathrm{~h}$ ) and $\mathrm{A} 3$ (48 and $72 \mathrm{~h}$ ). In the former case there was no mutual attraction, whereas in the latter case merger occurred at $79 \mathrm{~h}$. One can see no significant difference in the vorticity fields at $72 \mathrm{~h}$ in experiment $\mathrm{A} 1$ and $48 \mathrm{~h}$ in experiment $\mathrm{A} 3$. Both patterns are characterized by two well-separated vorticity maxima with small positive values between them. Thus it is hard to expect that in experiment A3 the vortices could merge. Note that in this case the distance between vortices even increased during the period of time from 24 to $48 \mathrm{~h}$. However, during the following 24-h period the combined vorticity field drastically changed. At $72 \mathrm{~h}$ (Fig. 19b) the amplitude of the positive vorticity between storms significantly increased, and the whole system of binary vortices was bounded by high vorticity values. We can thus conclude that the merger pro-

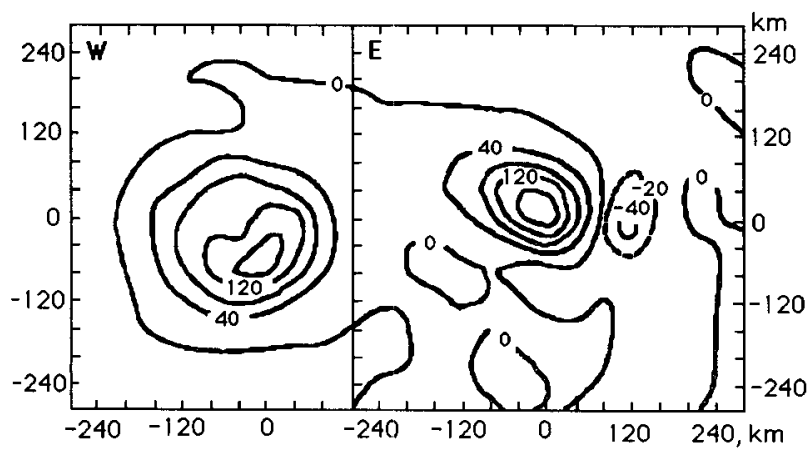

Fig. 18. Distribution of level $5(\sigma=0.5)$ vorticity $\left(10^{-5} \mathrm{~s}^{-1}\right)$ for experiment $\mathrm{Al}$ at $t=72 \mathrm{~h}$.
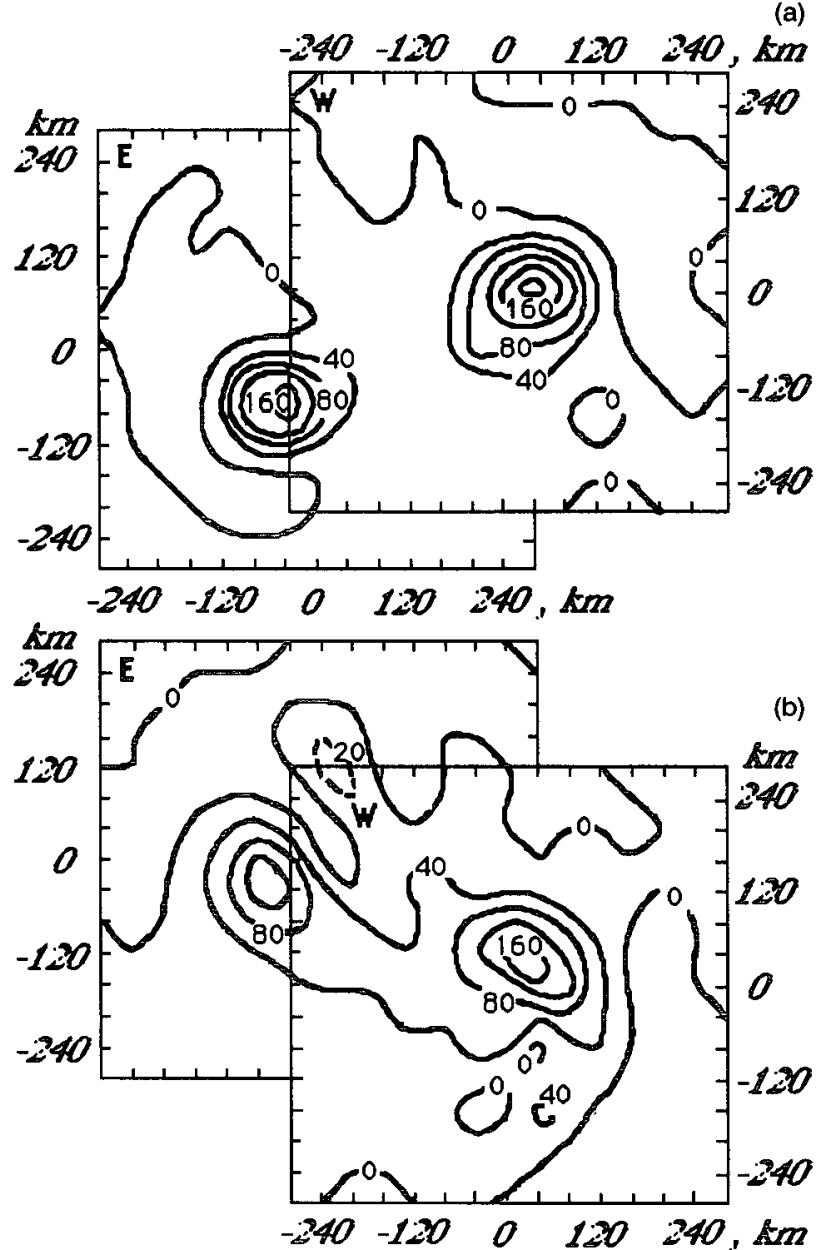

FIG. 19. As in Fig. 18 but for experiment $\mathrm{A} 3$ at $t=48 \mathrm{~h}$ (a) and at $t=72 \mathrm{~h}(\mathrm{~b})$.

cess is indeed accompanied by significant structural changes in the vorticity fields.

As mentioned previously, DeMaria and Chan (1984) suggest that mutual attraction of two vortices will depend on the sign of the radial vorticity gradient of the first vortex in the center of the second vortex. In order to check whether this assumption applies to the present experiments, we can compare the radial vorticity distributions in an experiment in which merger occurred with one in which storms separated. Let us consider, for example, the vorticity profiles along the line passing through the storm centers in experiments $\mathrm{AF}$ and $\mathrm{A} 4$ at $t=24 \mathrm{~h}$. These experiments are chosen because the structures (the radial distribution of tangential winds or vorticity) of the vortices $\mathrm{W}$ and $\mathrm{E}$ were quite similar. Figure 20 shows the combined vorticity profiles of both cyclones at the level $5(\sim 550 \mathrm{mb})$. In general, it is difficult to separate vorticity fields at the center of each storm. One possible way to accomplish this is to assume that the storms are axisymmetric and to turn over the vorticity profiles outside of the interactive area to 

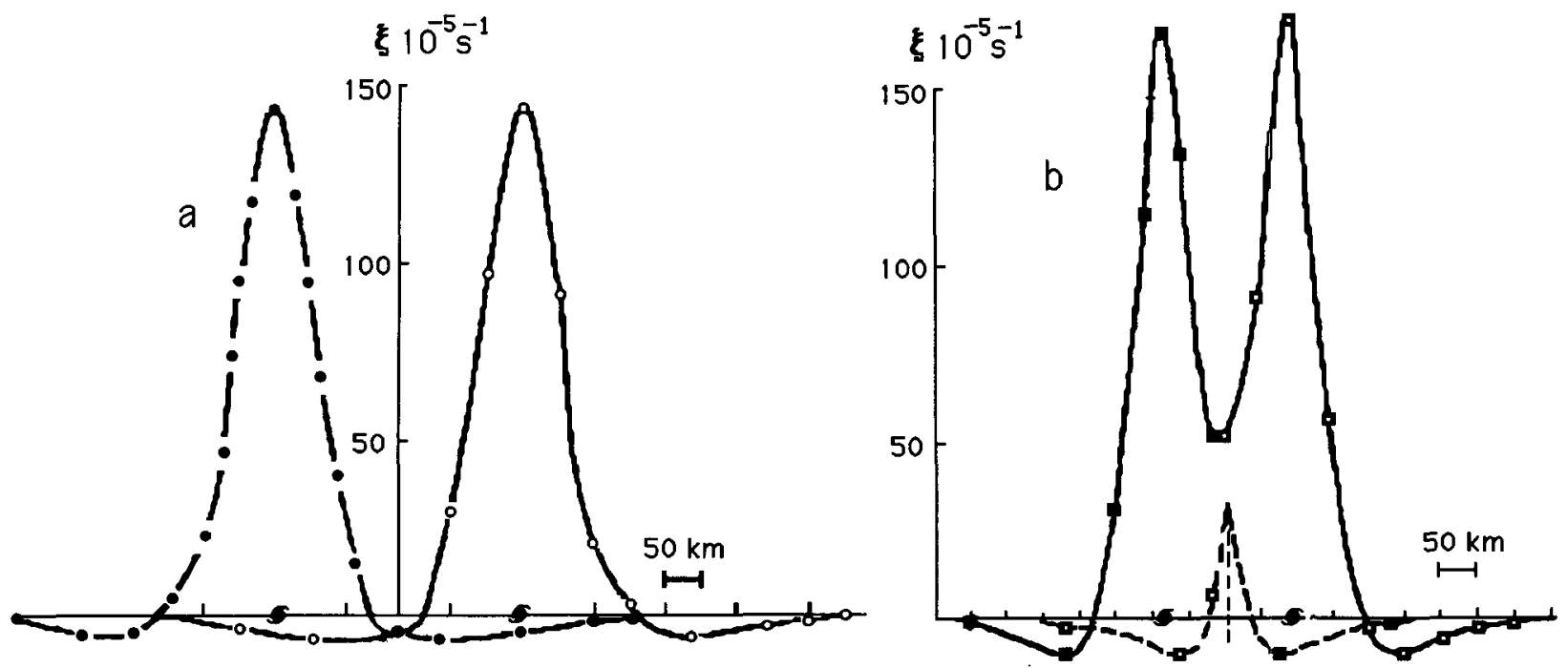

FIG. 20. Horizontal distribution of level $5(\sigma=0.5)$ vorticity along the axis connecting the storm centers in experiments $\mathrm{AF}$ (a) and $\mathrm{A} 4$ (b) at $t=24 \mathrm{~h}$.

the area between the storms. As one sees in Fig. 20, in both experiments AF and A4 the signs of the vorticity gradient of vortex $W$ at the center of vortex $E$ are positive. It follows that the separation distance is greater than $r_{c}$ and, according to DeMaria and Chan (1984), the storms must separate, though the merger occurred in experiment A4.

Recent numerical studies using nondivergent barotropic models would seem to help in clarifying the role of the vorticity structures in the process of attraction or repulsion of binary vortices. Pokhil et al. (1990) used two different tangential wind profiles

$$
\begin{gathered}
v(r)=1.65 V_{m}\left(\frac{r}{R}\right) \exp \left[-\frac{1}{2}\left(\frac{r}{R}\right)^{2}\right], \\
v(r)=3 V_{m}\left(\frac{r}{R}\right)\left[2+\left(\frac{r}{R}\right)^{3}\right]^{-1},
\end{gathered}
$$

where $R$ is the radius of maximum winds.

They found that the critical separation distance was $2.7 R$ for the profile (12) and $3.2 R$ for the profile (13). Chan (1993) performed a series of numerical experiments using the following wind profile

$$
v(r)=V_{m}\left(\frac{r}{R}\right) \exp \left\{\frac{1}{b}\left[1-\left(\frac{r}{R}\right)^{b}\right]\right\},
$$

where $b$ was set to either 1 or 0.7 . His results indicated that the critical separation distance was about $370 \mathrm{~km}$ for $b=1$ and $550 \mathrm{~km}$ for $b=0.7$, for a radius of maximum winds set to $R=100 \mathrm{~km}$.

The results of Pokhil et al. (1990) and Chan (1993) can be summarized as shown in Fig. 21. The figure depicts the wind and vorticity profiles of the binary vortices that are separated by the critical distances found in these studies. One sees that in all cases $d_{\mathrm{cr}}>r_{\mathrm{c}}$. Note another important feature: the critical separation distance in these experiments corresponds to nearly zero vorticity values between the vortices. Thus, the binary storms were attracted when positive vorticity values between the vortices occurred. This condition can be explained by the advection of the asymmetric vorticity of one vortex by the symmetric tangential flow of the other one. The situation resembles the case of developing beta gyres in an initially axisymmetric vortex on a beta plane. Figure 22 shows this effect schematically. Consider two axially symmetric vortices $W$ and $E$ on the same latitude. We use here the vorticity profile of the vortex $\mathrm{W}$ in experiment A4 (shown at the bottom of the figure). The tangential flow of the vortex $E$ transports the vorticity tail of the vortex W from point $C$ in the direction of point $D$ and from point $A$ in the direction of point $B$. As a result, asymmetric vorticity anomalies (secondary vorticity gyres) are developed to the north and south of the vortex E. Attraction or repulsion of binary vortices will be determined by the direction of the flow between these gyres. To decrease the separation distance, the vorticity anomalies to the south must be positive and their magnitudes must be greater than those of the vorticity anomalies to the north.

Figure 22 illustrates that for the barotropic framework, the suggested mechanism determining the attraction or repulsion between binary vortices is of the same nature as the mechanism causing the beta drift. The flow created between the beta gyres depends on advection of absolute vorticity by axisymmetric circulation at different radii, not just at one radius. However, the main contribution of the advection to formation of beta 


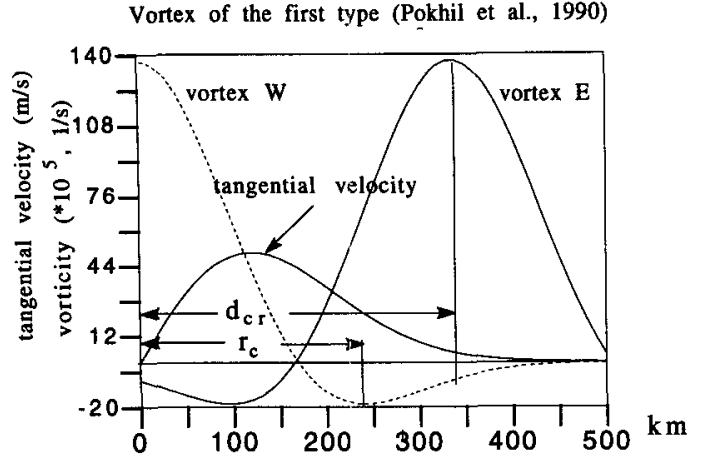

Vortex of the third type, $b=0.7$ (Chan, 1993)

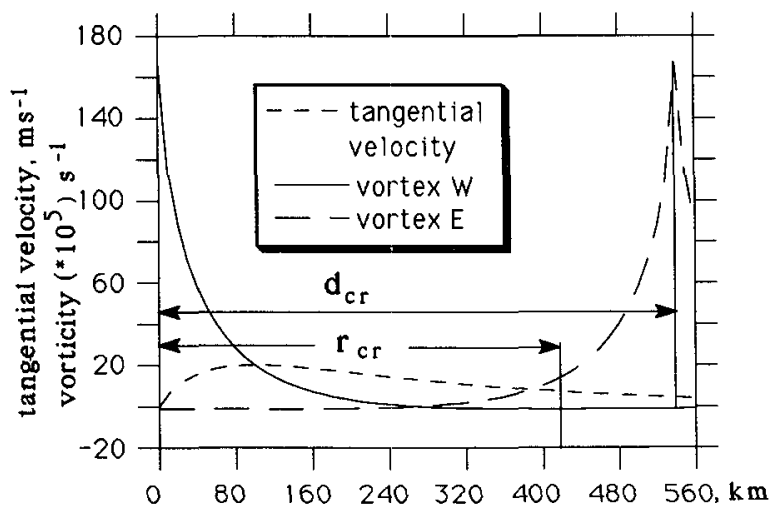

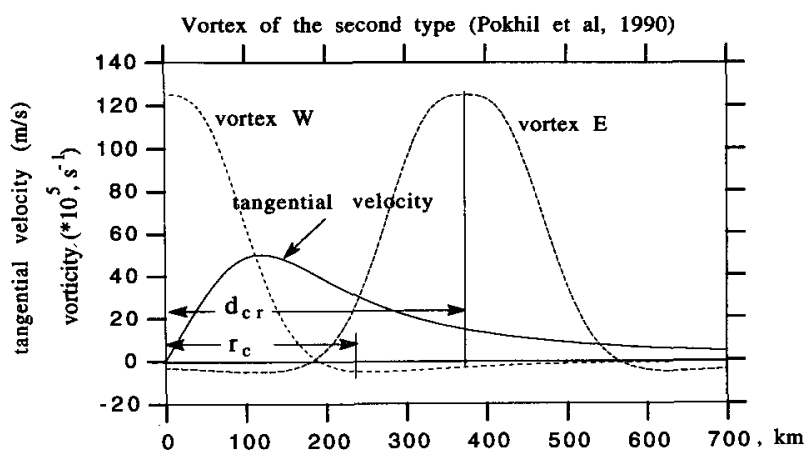

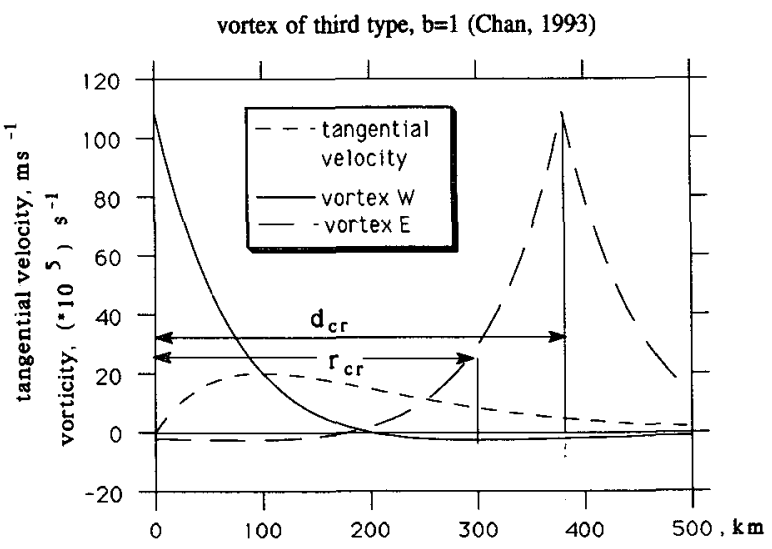

Flg. 21. The radial distributions of vorticity corresponding to (a) Eq. (12), (b) Eq. (13) (Pokhil et al. 1990), (c) Eq. (14) $b=0.7$, and (d) Eq. (14) $b=1$ (Chan 1993). Radius of maximum winds $100 \mathrm{~km}$. The binary vortices are separated by the critical distances found in the numerical experiments.

gyres takes place at the radii in the range of 200-300 $\mathrm{km}$. In case of mutual interaction of binary TC, the secondary flow at the center of vortex $\mathrm{E}$ is dependent on the vorticity distribution at all radii, not just at one radius. In other words, the flow at the center of vortex $\mathrm{E}$ is determined by an integral effect. We believe, however, that the main contribution to the flow at the center of vortex $\mathrm{E}$, determining its motion relative to the vortex $\mathrm{W}$, comes from the winds at the radii from 200 to $300 \mathrm{~km}$. Of course, for 3D tropical cyclones, the motion of binary storms is also determined by the contributions of the secondary circulation at different levels. The investigation of the latter mechanism is the subject of future work.

The attraction and repulsion of the binary storms in the experiments presented in this study seem to be in agreement with the above scheme. It can be seen in Fig. 20 that the attraction of the storms in experiment A4 and their repulsion in experiment AF are in a good agreement with the corresponding vorticity distributions. In these experiments, the vorticity of the vortex $\mathrm{W}$ is close to zero at the eastern periphery of the vortex $\mathrm{E}$ (point $A$ ), whereas the vorticity values are nearly the same at the northern (point $B$ ) and southern (point $D$ ) periphery of the vortex $E$.
It also should be noted that the interaction between the vortices in the pair may lead to the development of asymmetries in the vorticity fields. As a result, positive vorticity values between the storms could be found even in cases of repulsion (see, for example, experiment A1, Fig. 18). Hence, the presence of small positive vorticity values at certain levels between 3D asymmetric vortices may appear to be insufficient for storm attraction. The values should be greater than a certain threshold that seems hard to determine a priori.

Although the mechanisms of attraction or repulsion of the binary storms in a baroclinic model can be partially explained using barotropic model simulations, there is one important difference in the two models that should be stressed. In a barotropic model there is no mechanism that can prevent the shape of one of the interacting vortices from stretching and distorting in the horizontally sheared flow created by the other one. Therefore, the maximum vorticity values of the vortices usually decrease rapidly as the storms approach closer to each other (e.g., Pokhil et al. 1990; Pokhil 1991 ). In contrast, vorticity structures in a baroclinic model tend to maintain their shape and intensity even at rather small separation distances (Fig. 19). The latter effect may substantially modify the structure of the vor- 


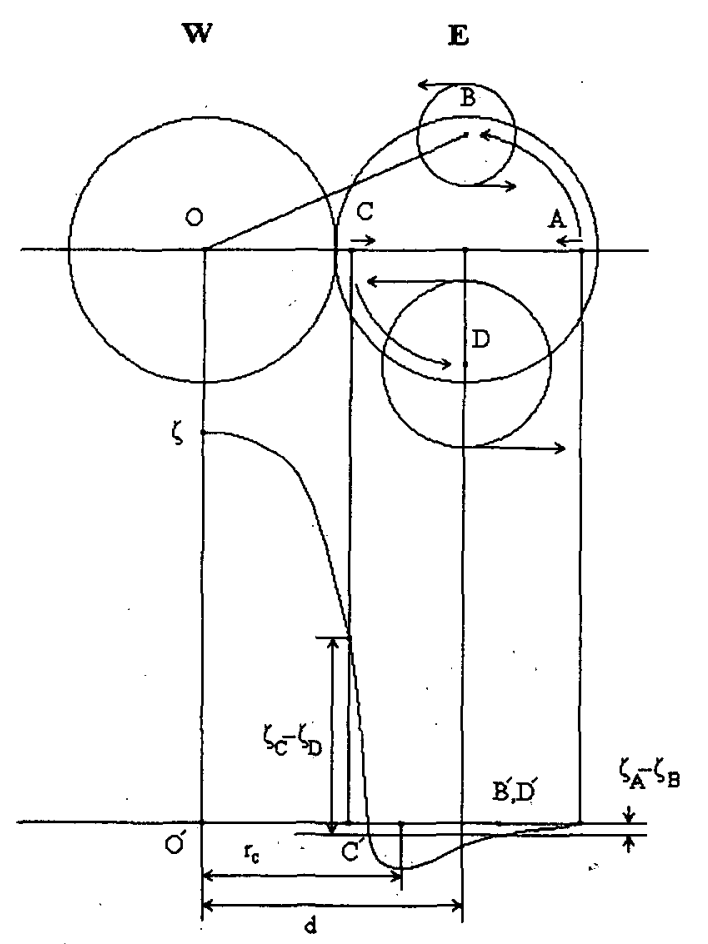

FIG. 22. A scheme of a mutual tangential vorticity advection influencing the separation distance between two binary vortices. In the bottom of the figure the vorticity profile of the vortex $\mathrm{W}$ in experiment $A 4$ is shown.

ticity distribution between the storms and therefore influence the merger process.

In order to investigate the possible role of baroclinic effects involved in the interaction of binary vortices, additional numerical integrations were carried out using a barotropic model with an artificial sink of mass. The model is a vertically integrated version of the $3 \mathrm{D}$ model described in section 2 on the $f$ plane. In the experiments performed, a sink of mass was introduced in the central areas of the barotropic vortices as $125[1$ $\left.+\cos \left[\pi r(3 s)^{-1}\right]\right\}$ for $r<3 s$, where $s=50 \mathrm{~km}$.

In the first set of experiments we compared the integrations with and without the mass sink using the wind profile (14) for $b=1, R=100 \mathrm{~km}$, and $V_{m}=60$ $\mathrm{m} \mathrm{s}^{-1}$. It turned out that the vortices initially separated at $350 \mathrm{~km}$ merged in the experiment with no sink of mass [in agreement with Chan (1993)] but moved away in the experiment with sink of mass. Inspection of the vorticity fields in these simulations indicates that in the latter experiment the vortices were much more concentrated than the ones in the former experiment. The analogous experiments performed using the wind profile (12) $\left(R=180 \mathrm{~km}, V_{m}=60 \mathrm{~m} \mathrm{~s}^{-1}\right)$ gave qualitatively similar results. That is, for the initial separation distance of $450 \mathrm{~km}$, the vortices repelled (merged) with (without) a sink of mass. We can thus conclude that baroclinic effects tend to reduce the critical separation distance.

Ritchie and Holland (1993) and Holland and Dietachmayer (1993) suggested another mechanism determining merger or repulsion of tropical storms. This mechanism is based on the competition effects of strain imposed on each vortex by the sheared potential flow of the other vortex and the tendency toward rotational symmetry in each vortex patch. The baroclinic effects mentioned increase the tendency toward rotational symmetry and, according to Ritchie and Holland (1993) and Holland and Dietachmayer (1993), should lead to a decrease in the critical separation distance. This is consistent with results of our computations. Comparison of the mechanisms of binary tropical cyclones interaction discussed in this paper with that suggested in the studies by Ritchie and Holland (1993) and Holland and Dietachmayer (1993) awaits further investigation.

Another effect that might also influence the interaction of binary storms in a baroclinic model is the irrotational component of the storm circulation (wind flow divergence). This effect, first identified by Chang $(1983,1984)$, may enhance the attraction process due to the advection of the vorticity field of one vortex by the mean inward radial flow of the other vortex. The role of the wind convergence in a merger of convective clouds has been demonstrated recently by Shapiro and Kogan (1995). They found that the initially separated convective clouds began to attract by the wind convergence, and the distance between them decreased faster at the cloud base where the inflow radial velocity had a maximum. Shapiro and Kogan also identify a critical separation distance between the clouds based only on the irrotational wind components.

We finally note that irrotational circulation might also modify the distribution of the combined vorticity field between the binary vortices and therefore influence the barotropic mechanism discussed previously. Thus, it is generally difficult to distinguish the contributions of the baroclinic and barotropic mechanisms to the binary storm interaction in 3D simulations because of the synergistic effect of both mechanisms. A detailed analysis of these effects will be the subject of future research.

\section{Summary and conclusions}

In the present study, the evolution and motion of binary tropical cyclones was investigated using a coupled tropical cyclone-ocean movable nested-grid model. The model consists of eight-layer atmospheric and three-layer ocean primitive equation models. There are five meshes in the atmospheric model. The outermost domain is motionless, while the four internal meshes ( two telescopically nested meshes for each cyclone) move with storm centers. The ocean model uses a uniform mesh that is similar to the finest meshes in the atmospheric model. 
In a set of numerical experiments, a pair of axisymmetric vortices of equal structure and intensity were initially separated by specified distances. The ocean was assumed to be horizontally uniform and motionless. The environmental atmospheric and oceanic conditions were set in such a way as to allow the vortices to rapidly develop to hurricane intensities.

Numerical simulations revealed that the binary storms evolve on a beta plane in a highly asymmetric manner in the course of their interaction. It turned out that the storm initially located to the west (storm W) always intensifies faster than the one initially located to the east (storm $\mathrm{E}$ ). This effect is related to the fact that during the initial period (about $36 \mathrm{~h}$ ) of storm interaction the mutual rotation of the storms causes storm $\mathrm{W}$ (storm E) to move southeast (northwest). Hence, storm $\mathrm{W}$ moves against, whereas storm $\mathrm{E}$ moves along, the beta drift. As a result, storm W moves slower than storm $\mathrm{E}$ shortly after the beginning of the integration. This effect is speculated to be the trigger for the asymmetric development of the binary storms for the current set of experiments. This is because the rate of storm deepening in the present model is quite sensitive to the storm translation speed: slower moving storms intensify more rapidly. It should be noted, however, that there is little conclusive observational evidence indicating that the development of tropical cyclones is dependent on the magnitude of mean basic flows. Nevertheless, such a tendency seems to agree well with other numerical studies (e.g., Falkovich et al. 1995; Bender et al. 1993).

Once the initial asymmetry is established by the above mechanism, it continues to grow further during the mutual storm rotation. This is because the tangential circulation of the one vortex is a steering flow for the other one in the vortex pair; and therefore, the more intense storm generates a stronger rotation. As a result, the storm moving in a stronger steering flow deepens more slowly. Another important effect that also contributes to the storm growth asymmetry is related to the fact that the more intense storm is accompanied by a stronger vertical wind shear. Therefore, the other storm is moving in a highly sheared steering flow that is known to impede cyclone evolution (e.g., Gray 1979; Kurihara and Tuleya 1981; Shapiro 1992).

Whether two interacting cyclones will attract or repel is found to be sensitive to the initial "critical" separation distance. This distance is determined by the structure and intensity of the storms. Both the rotational (vorticity) and irrotational (divergence) components of the storm circulations seem to contribute to the process of merger. Analysis of the previous studies using nondivergent barotropic models suggests that one of the possible conditions for storm merger could be positive vorticity values between the vortices in the combined vorticity field. The physical reasoning for this condition is explained by advection of the asymmetric vorticity of one vortex by a symmetric tangential flow of another vortex. Analysis of the 3D model simulations in this study indicates that this condition is probably necessary but not sufficient for a storm merger. In one of the experiments in which storms moved away, a small magnitude of positive vorticity between them was observed.

The contribution of the baroclinic effects to the storm merger can be twofold. On the one hand, supplemental experiments with a barotropic model, including a mass sink, indicate a decrease of the critical separation distance in comparison with the experiments with no mass sink. Analysis of the results suggests that in the former experiments each storm was able to maintain the axisymmetric shape of the vorticity field against stretching and distortion for closer separation distances. In this sense, the baroclinic effects decrease the critical separation distance. On the other hand, the radial-vertical circulation in the inflow layer associated with a stronger tropical cyclone might contribute to the storm attraction and therefore cause an increase of the critical separation distance. Thus, it is very difficult to evaluate the role of the baroclinic effects in the process of storm merger. It is even more difficult to separate the contributions of the rotational and irrotational components of the storm circulations to this process.

Interaction with the ocean had a significant effect on the evolution, intensity, and tracks of the binary storms for the current set of experiments. The importance of the decrease of sea surface temperature caused by the ocean response to the surface wind stress has been highlighted recently by Khain and Ginis (1991), Bender et al. (1993), and Falkovich et al. (1993, 1995). However, this effect is found to be especially important for the binary storms' behavior. The reasons for this can be summarized as the following.

The storm weakening appeared to be stronger compared to single tropical cyclones since the binary storms are crossing the tracks of each other during the interaction. A reduction of tropical cyclone strength in the pair, in turn, causes slower mutual rotations (storm translation speeds). Accordingly, this leads to significant changes in storm tracks.

It is also found that the ocean coupling decreases the asymmetry of the intensity and strength of binary storms in comparison to noncoupled experiments. This is because the more intense and slower-moving tropical cyclones produce larger sea surface cooling that, in turn, induces stronger storm weakening. As a result of the structural changes of the binary storms due to the ocean coupling, the critical separation distance is also modified.

Finally, we must note that the numerical experiments presented in this study are performed for highly idealized conditions, assuming no basic flow in the atmosphere and a horizontally homogeneous and quiescent ocean. It can be speculated that the influence of the ocean response for more realistic cases (such as, for 
example, in the presence of a basic flow ) may modify some of our conclusions. However, the basic mechanisms revealed in this study hopefully will not change drastically for real environment conditions. An extensive investigation of this topic awaits further research.

Acknowledgments. This research was partially supported by the United States-Israel Binational Science Foundation under Grant 9200350.

\section{REFERENCES}

Bender, M. A., I. Ginis, and Y. Kurihara, 1993: Numerical simulations of hurricane-ocean interaction with a high resolution coupled model. J. Geophys. Res., 98 (D12), 23 245-23 263.

Black, P. G., 1983: Ocean temperature change induced by tropical cyclones. Ph.D. dissertation, The Pennsylvania State University, 278 pp.

Brand, S., 1970: Interaction of binary tropical cyclones of the western North Pacific Ocean. J. Appl. Meteor., 9, 433-441.

Chan, J. C.-L., 1993: The interaction of binary vortices in a barotropic model: Tropical Cyclone Disasters, J. Lighthill, Z. Zhemin, G. J. Holland, and K. Emanuel, Eds., Peking University Press, 296-302.

Chang, S. W., 1979: The response of an axisymmetric model of tropical cyclone to local variations of sea surface temperature. Mon. Wea. Rev., 107, 662-666.

- 1983: A numerical study of the interactions between two tropical cyclones. Mon. Wea. Rev., 111, 1806-1817.

_ 1984: Reply. Mon. Wea. Rev., 112, 1646-1647.

Deardorff, J. W., 1972: Parameterization of the planetary boundary layer for use in general circulation models. Mon. Wea. Rev., 100, 93-106.

- 1983: A multi-limit mixed layer entrainment formulation. $J$. Phys. Oceanogr., 13, 988-1002.

Dèaria; M., and J. C. L. Chan, 1984: Comments on "A numerical study of the interactions between two tropical cyclones." Mon. Wea. Rev., 112, 1643-1645.

Dong, K., and C. J. Neumann, 1983: On the relative motion of the binary tropical cyclones. Mon. Wea. Rev., 111, 945-953.

Elsberry, R. L., 1993: Tropical cyclone motion. Global Perspectives on Tropical Cyclones.

Emanuel, K. A., 1989: The finite-amplitude nature of tropical cyclogenesis. J. Atmos. Sci., 46, 3431-3456.

_ 1991: The theory of hurricanes. Annu. Rev. Fluid. Mech., 23, 179-196.

Falkovich, A. I., 1986: A nested-grid scheme for predicting isolated vortex motion in a barotropic atmospheric model. Sov. Meteor. Hydrol., No. 9, 35-41.

- 1988: Adaptation of meteorological fields in a baroclinic model. Sov. Meteor. Hydrol., No. 9, 29-42.

- - , and A. V. Kulakov, 1986: On prediction of single vortex motion. Sov. Meteor. Hydrol., Nó. 8, 58-65.

- A. P. Khain, and I. D. Ginis, 1992: Studying of the development and motion of tropical cyclones by use of a coupled ocean-atmosphere model. Sov. Meteor. Hydrol., No. 2, 2339.

- and - 1993: The evolution and motion of two interacting tropical cyclones in a coupled model of the atmosphere and the ocean with movable nested grids. Preprints, 20th Conf. on Hurricanes and Tropical Meteorology. San Antonio, TX, Amer. Meteor. Soc., 374-377.

- - - and -1995 : The influence of the air-ocean interaction on the development and motion of a tropical cyclone: numerical experiments with a triply nested model. Meteor. Atmos. Phys.; in press.

Fiorino, M., and R. L. Elsberry, 1989: Some aspects of vortex structure related to the tropical cyclone motion. J. Atmos. Sci., 46, 975-990.
Fujiwhara, S., 1921: The natural tendency towards symmetry of motion and its application as a principle in meteorology. Quart. $J$. Roy. Meteor. Soc., 47, 287-293.

Ginis, I., and K. Z. Dikinôv, 1989: Modelling of the typhoon Virginia (1978) forcing on the ocean. Sov. Meteor. Hydrol., No. 7, 5360.

- and G. Sutyrin, 1995: Hurricane-generated depth-averaged currents and sea surface elevation. J. Phys. Oceanogr., in press.

Gray, W. M., 1979: Hurricanes: Their formation, structure and likely role in the tropical circulation. Meteorology over Tropical Oceans, Roy. Meteor. Soc. 105, 155-218.

Holland, G. J., and G. S. Dietachmayer, 1993: On the interaction of tropical-cyclone-scale vortices. III: Continuous barotropic vortices. Quart. J. Roy. Meteor. Soc., 119, 1381-1398.

Ivanov, V. N., and V. D. Pudov, 1977: Structure of the thermal wake of typhoon Tess in the ocean and estimation of the certain energy-exchange parameters under storm condition. Typhoon-75, Gidrometizdat, Vol. 1, 66-82.

Jordan, C. L., 1958: Mean soundings for the West Indies area. $J$. Meteor., 15, 91-97.

Khadekar, M. L., and G. V. Rao, 1971: The mutual interaction of multiple vectors and its influence on binary and single tropical vortex storms. Mon. Wea. Rev., 99, 840-846.

Khain, A. P., 1979: The 12-level axisymmetric numerical tropical cyclone model. Sov. Meteor. Hydrol., No. 10, 23-37.

, 1980: Reaction of axisymmetric tropical cyclone to variation of the sea surface temperature and evaporation. Sov. Meteor. Hydrol., Vol. 10, 59-63.

_, 1984: Mathematical Modelling of Tropical Cyclones. Gidrometizdat, $247 \mathrm{pp}$.

_ 1988: A three-dimensionăl numerical model of a tropical cyclone with allowance for beta-effect. Atmos. Oceanic Phys., 24, 266-271.

- and I. D. Ginis, 1991: The mutual response of a moving tropical cyclone and the ocean. Beitr. Phys. Atmos., 64/2, 125-142.

Kurihara, Y., and R. E. Tuleya, 1974: Structure of a tropical cyclone developed in a three-dimensional model. J. Atmos. Sci., 31, $893-919$.

$\longrightarrow$, and -1981 : A numerical simulation study on the genesis of a tropical storm. Mon. Wea. Rev., 109, 1629-1653.

— G. J. Tripoli, and M. A. Bender, 1979: Design of a movable nested-mesh primitive equation model. Mon. Wea. Rev., 107, 239-249.

Lander, M., and G. J. Holland, 1993: On the interaction of tropicalcyclone-scale vortices. I: Observations. Quart. J. Roy. Meteor. Soc., 119, 1347-1361.

Leipper, D. L., 1967: Observed ocean conditions and hurricane Hilda, 1964. J. Atmos. Sci., 24, 182-196.

Marchuk, G. I., 1982: Methods of Numerical Mathematics. Springer Verlag, $510 \mathrm{pp}$.

Neumann, C. J., 1981: Trends in forecasting the tracks of Atlantic tropical cyclones. Bull. Amer. Meter. Soc., 62, 1473-1485. , 1993: Global Overview. Global Guide to Tropical Cyclone Forecasting. World Meteorological Organization, 1.1-1.56.

Orlanski, I., 1976: A simple boundary conditions for unbounded hyperbolic flows. J. Comput. Phys., 21, 251-269.

Pekelis, E. M., and D. Y.. Pressman, 1982: Numerical implementation of radiation boundary conditions and their applications to the conditions of "open boundaries" for numerical integration of the equations of convection. Proc. USSR Hydrometeorological Center, 249, 74-95 (in Russian).

Pokhil, A. E., 1990: On interaction between tropical cyclones in the Pacific Ocean. Sov. Meteor. Hydrol., No. 6, 60-67.

- 1991: On the formation and dissipation of secondary vortices during the interaction of two tropical cyclones (numerical experiment). Sov. Meteor. Hydrol., No. 9, 34-41.

, I. G. Sitnikov, V. A. Zlenko, and I. V. Polyakova, 1990: A numerical study of interacting atmospheric vortices. Sov. Meteor. Hydrol., No. 4, 21-28. 
Pressman, D. Y., 1968: Integration of complete equations of shortrange weather forecasting according to explicit differential schemes. Tr. Hydrometeorological Center USSR, 16.

Price, J. F., 1981: Upper ocean response to a hurricane. J. Phys. Oceanogr., 11, 153-175.

Ramage, C. S., 1974: Interaction between tropical cyclones and the China Sea. Weather, 27, 484-494.

Raymond, W. H., and H. L. Kuo, 1984: A radiation boundary condition for multi-dimensional flows. Quart. J. Roy. Meteor. Soc., $110,535-551$.

Ritchie, E. A., and G. J. Holland, 1993: On the interaction of tropicalcyclone-scale vortices. II: Discrete vortex patches. Quart. J. Roy. Meteor. Soc., 119, 1363-1379.

Rosenthal, S. L., 1978: Numerical simulation of tropical cyclone development with latent heat by the resolvable scales. I. Model description and preliminary results. J. Atmos. Sci., 35, 258271.

Shapiro, L. J., 1992: Hurricane vortex motion and evolution in a three-layer model. J. Atmos. Sci., 49, 140-153.

,-- , and Y. Kogan, 1995: Simulation of cloud mergers in a three-dimensional model. Preprints, Conf. on Cloud Physics, Dallas, TX, Amer. Meteor. Soc., 312-317.

Shay, L. K., P. G. Black, A. J. Mariano, J. D. Hawkins, and R. L. Elsberry, 1992: Upper ocean response to Hurricane Gilbert. $J$. Geophys. Res., 97, $20227-20248$.

Shuman, F. G., and J. B. Hovermale, 1968: An operational six layer primitive equation model. J. Appl. Meteor., 7, 525-547.

Tuleya, R. E., and Y. Kurihara, 1982: A note on the sea surface temperature sensitivity of a numerical model of tropical storm genesis. Mon. Wea. Rev., 110, 2065-2071. 